\title{
Estimation of Non-Normal Process Capability Indices
}

\author{
Huda H.M. Tayeb \\ hudaaldahir@gmail.com
}

Ban Gh.O. Al-Ani

drbanalani@uomosul.edu.iq

Department of Statistic

College of Computer Science and Mathematics, University of Mosul, Mosul, Iraq

Received on: 10/07/2020

Accepted on: 15/08/2020

\begin{abstract}
The important and common applications of process capability indices (PCI's) is the process capability ratio $C_{p}$ and the process capability index $C_{p k}$, and the use of these measures in practical applications is based on the assumption that the process is under statistical control and that the outputs of that process follow the normal distribution. In practice, however, there are many cases in which the outputs of the process are not follow normal distribution, in such cases, calculating PCI's will lead to misleading results. In this paper, the non-normal production process capability was evaluated. The evaluation process was conducted using four methods: The first method involved the use of Box-Cox power transformation to normal distribution of data and then calculating indicators of the capability process by traditional methods. The second method is by using the weighted variance method, and the third method is by using the Clements' method based on the percentiles calculation of data, and the fourth method is based on the use of the Darling-Anderson goodness of fit test to accommodate some of the probability distributions of the original data, this test shows that the log-normal distribution is the most appropriate distribution for the data The results showed that although the process is stable and under statistical control, it is not capable based on the value of the process capability index that did not exceed $(65 \%)$, as the percentage defect units, which is outside the specifications, is large and located between (3.6\%) and (13.4\%). The results also showed that there is a convergence in the results of the PCI's calculated by all methods, and that the Clements' method is the best method as it gives the highest values for the PCI's. The research recommended studying and calculating other PCI's like, $C_{s}, C_{p m}$, and $\operatorname{CMA}(\tau, \mathrm{v})$. One of the recommendations for future research and studies is to do a theoretical study to compare the mentioned methods and choose the best ones based on some statistical criteria.
\end{abstract}

Keywords: Process Capability Indices, Clements' Method, Weighted Variance Method, Box-Cox Transformation.

$$
\begin{aligned}
& \text { تقدير مؤشرات مقدرة عملية غير خاضعة للتوزيع الطبيعي }
\end{aligned}
$$

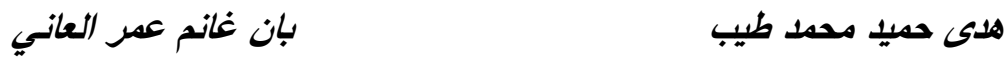

$$
\begin{aligned}
& \text { قسم الإحصاء والمعلوماتية، كلية علوم الحاسوب والرياضيات } \\
& \text { جامعة الموصل، الدوصل، العراق }
\end{aligned}
$$

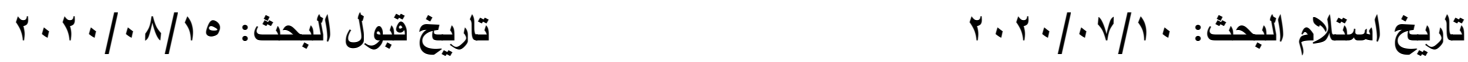




\section{الملخص}

من مقاييس مقدرة العملية المهمة والثائعة التطبيق، نسبة مقدرة العملية C ومؤشر مقدرة العملية CP ويستند استخدام هذه المقاييس في التطبيقات العملية إلى افتراض أن تكون العملية واقعة تحت السيطرة الإحصائية،

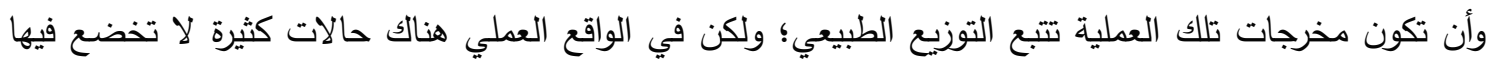
مخرجات العملية للتوزيع الطبيعي، في مثل هذه الحالات فانَّ حساب مؤشرات مقدرة العملية سيقود إلى نتائج مضللة، في هذا البحث قيمت مقدرة عملية إنتاجية لا تتبع التوزيع الطبيعي، جرت عملية التيقات فيتيم باستخدام أربعة أساليب، الأسلوب الأول: تضمَّن استخدام تحويل القوى Box-Cox لتوفير خاصية التَّزيع الطبيعي للبيانات ثم حساب مؤشرات مقدرة العملية بالطرائق التقليدية، الأسلوب الثاني: هو باستخدام طريقة التباين الموزون، والأسلوب

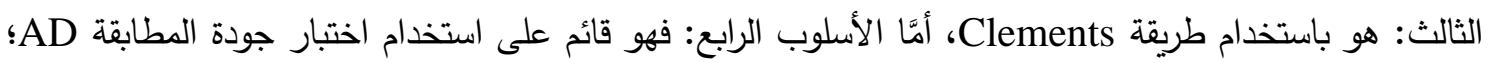

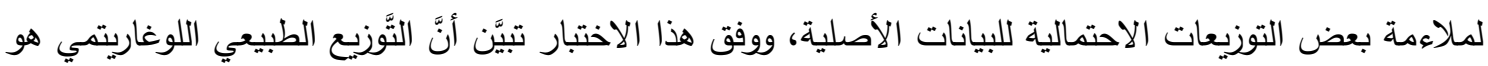

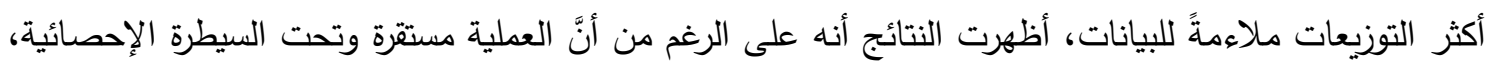

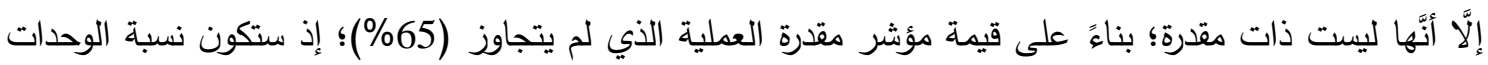

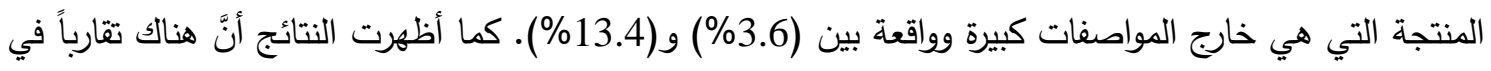
نتائج مؤشرات مقدرة العملية المحسوبة بجميع الطرائق، وأنَّ طريقة Clements أفضل الطرائق؛ كونها تعطي أعلى

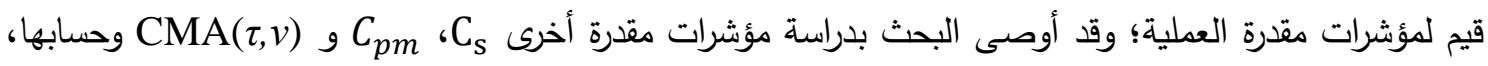

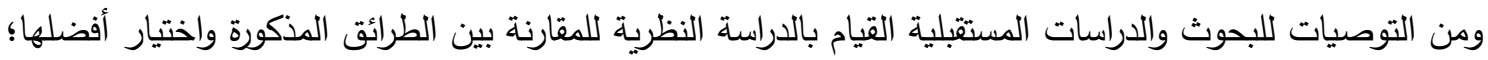
بناءً على بعض المعايير الإحصائية. الكلمات المفتاحية: مؤشرات مقدرة العملية، طريقة كليمنتس، طريقة التباين الموزون، تحويل بوكس -كوكس

\section{1. المقدمة:}

لقد أصبح موضوع مقدرة العملية من المواضيع الإحصائية المهمة التي تضمن جودة المنتجات الصناعيّة والرقابة عليها، وأصبح بمثابة القاسم المشترك لمختلف الاهتمامات الاقتصادية والإدارية والصناعيّة في كافة الدول النامية والمتقدمة على حد سواء؛ إذ يحظى بالعناية والاهتمام لقضيّة الجودة وهذا نتيجة منطقية لوعي متزايد انتهت إليه

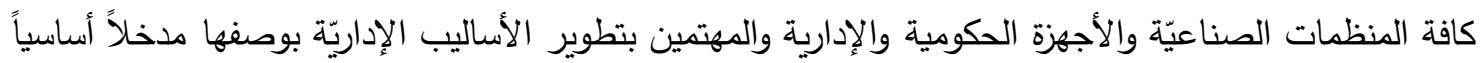
لمواجهة التحديّات المستقبليّة المُرتبطة بالهُحيط التتافسي الذي تتشط فيه المنظمة مما أدّى إلى تطوير مؤشرات مقدرة

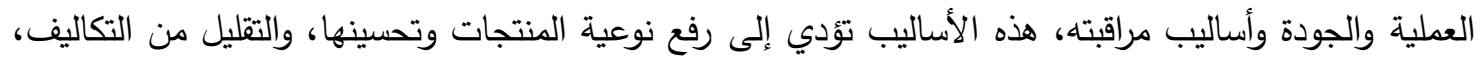
الثيء الذي يمكنها من الحفاظ على الحصة السوقية وكسب رضا الزبون، فمع ارتفاع مستويات التعليم والوعي أصبح

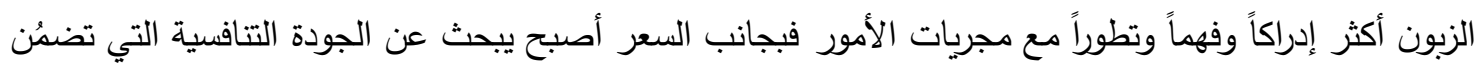
تلبية احتياجاته ومتطلباته.

تتحور مُشكلة البحث الأساسية في كيفية معالجة العمليات التي لا تتبع خصائصها التوزيع الطبيعي لتوافر

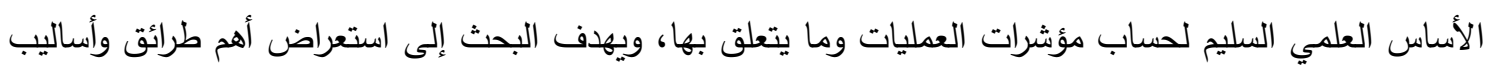

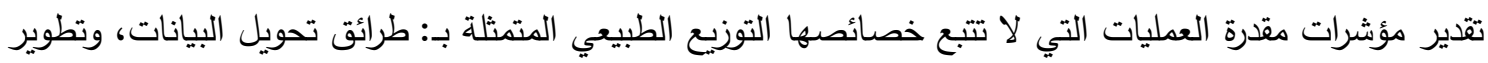

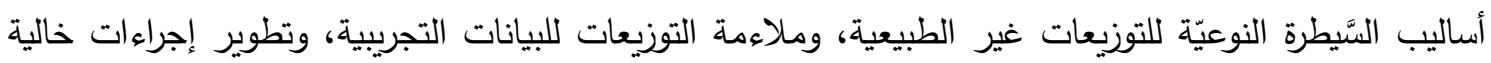


من التوزيعات، واستتاج مؤشرات جديدة لمقدرة العملية غير الطبيعية، ثُّ بيان كيفية تطبيقِ بعضِ هذه الطرائق من خلال تتاول الحالة الدراسيّة للبحث، وعمل مقارنة بها لاختيار أفضلها التي تعطي أفضل مؤشرات مقدرة للعملية.

2. مؤشرات مقدرة العملية: Process Capability Indices (PCI's)

مؤشرات مقدرة العمليات هي مقاييس تستعمل في تحديد العلاقة بين تثتت العملية وحدود المواصفات،

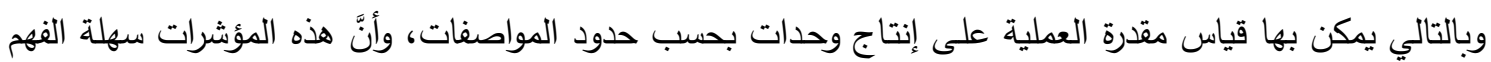
وبسيطة التطبيق تعبر عن أداء العملية، وخلاصة ما يحدث في الإنتاج بقيمة عددية.

1.2. نسبة مقدرة العملية: Process Capability Ratio (PCR)

اعتتى الكثير من الباحثين بوضع مقاييس عديدة وتطويرها؛ لقياس العملية الإنتاجية سُميّت بمؤشرات مقدرة العمليات PCI's فكان مؤشر نسبة مقدرة العملية C أول مؤشر وضعه Juran في عام (1974) وهو الأكثر ظهوراً في الدراسات، وعرّفه بأنه يُقارن مقدار انتشار العملية مع حدود المواصفات ويحسب بالصيغة الآتية: $C_{p}=\frac{U S L-L S L}{6 \sigma}$

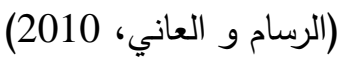

Upper Specifications Limit تإذ إنّ USL تمثل الحد الأعلى للمواصفات القياسية Lower Specifications Limit تمثل الحد الأدنى للمواصفات القياسية LSL

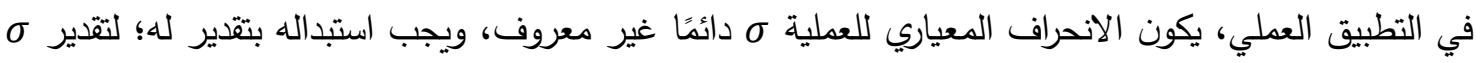
نستخدم عادةً الانحراف المعياري للعينة S أو R/d (عند استخدام لوحات السيطرة للمتغيرات في دراسة المقدرة). $\hat{C}_{p}=\frac{U S L-L S L}{6 \hat{\sigma}}=\frac{U S L-L S L}{6\left(\bar{R} / d_{2}\right)}$ ينتج عن ذلك تقدير لـ هو عاده $\bar{R}=\frac{1}{k} \sum_{j=1}^{k} R_{j} \quad k$ :number of samples إذ R يمثل متوسط مديات العينات أي إنَّ: و $d_{2}$ .(Montgomery, 2013) إن استخدام المقام (6б) في المعادلة (2) يعود إلى الفكرة نفسها التي قادت شيوارت إلى استخدام ثلاثة

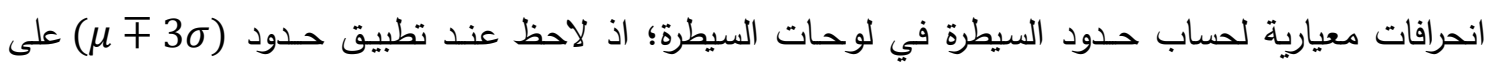
البيانات أنها تعطي نتائج جيدة من حيث التطبيق وانجاز حدود ذات تأثير، وهذا يدل على أنَّ (99.73\%) من

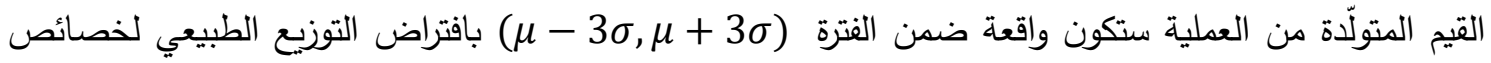

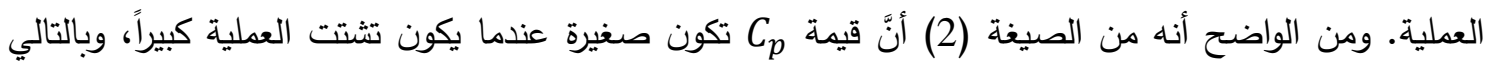
تكون العملية ذات مقدرة منخفضة. 


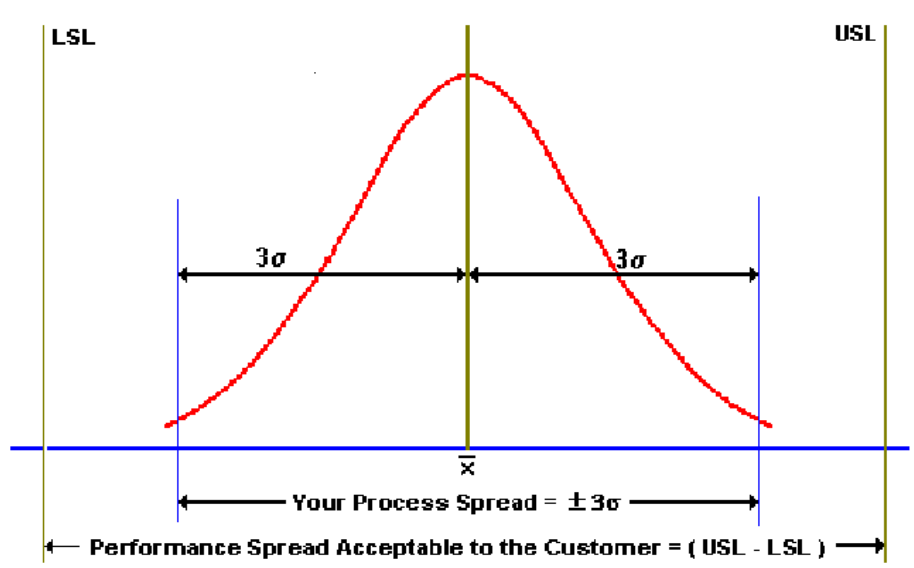

الثكل (1): التوزيع الطبيعي ومقدرة العملية

نسبة الوحدات المعيبة:

من السهل تطوير صيغة لتقدير النسبة المئوية للعيوب الناتجة عن العملية من قيمة C بافتراض أنَّ التوزيع

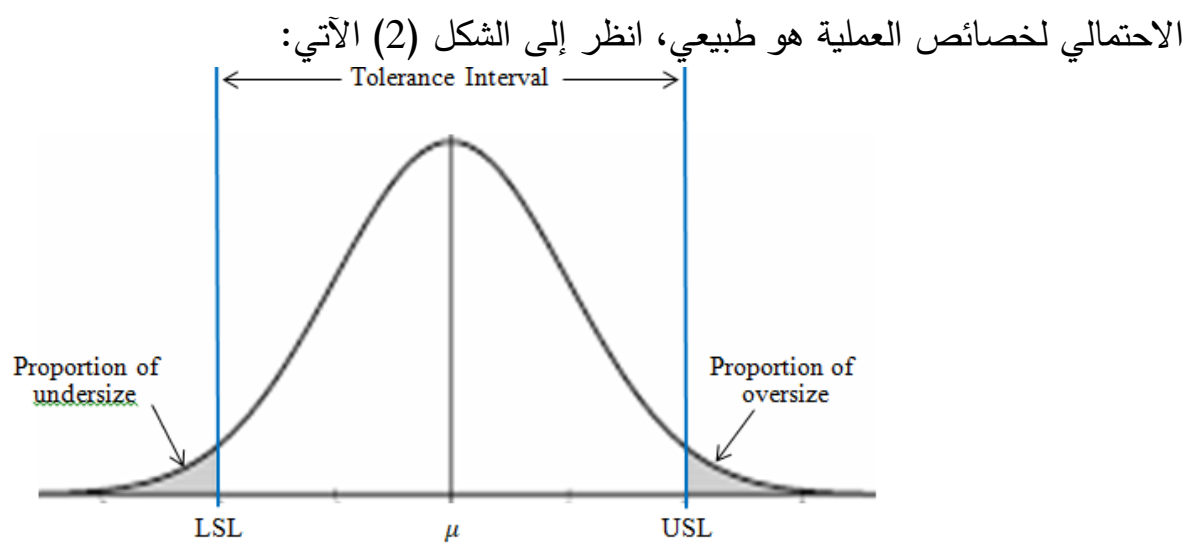

الثكل (2): نسب عدد الوحدات المعيبة (غير المطابقة للمواصفات)

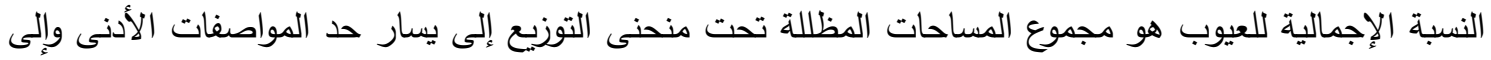
$p=\operatorname{Prob}(X<L S L)+\operatorname{Prob}(X>U S L)$

$$
=P\left(Z<\frac{L S L-\mu}{\sigma}\right)+P\left(Z>\frac{U S L-\mu}{\sigma}\right)
$$

إذا كان متوسط التوزيع يساوي نقطة منتصف فترة السماح عندئذ تكون مساحة المنطقتين المظللتين متساويتين $p=2 P\left(Z<\frac{L S L-\mu}{\sigma}\right) \quad$ or $\quad 2 P\left(Z>\frac{U S L-\mu}{\sigma}\right)$

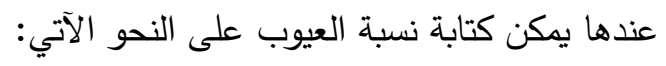
$\mu=\frac{1}{2}(L S L+U S L)$

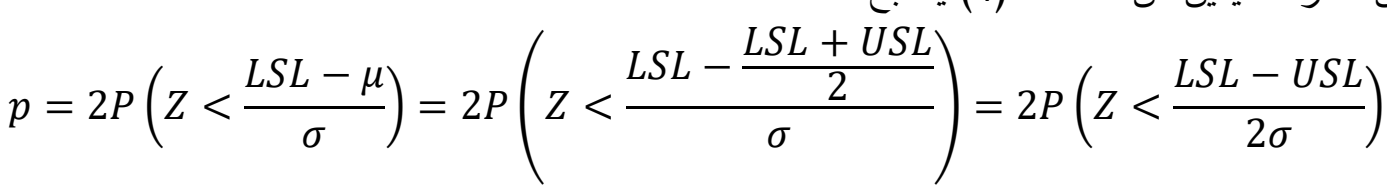


وبتعويض المعادلة (1) في المعادلة الأخيرة نحصل على:

$p=2 P\left(Z<\frac{-6 \sigma C_{p}}{2 \sigma}\right)=2 P\left(Z<-3 C_{p}\right)$

$p=2 P\left(Z>\frac{U S L-\mu}{\sigma}\right)=2 P\left(Z>3 C_{p}\right)$

وبالمثل فإنَّ:

عليه يمكن كتابة المعادلة (4) بالثكل الآتي:

$p=2 P\left(Z<-3 C_{p}\right)$ or $2 P\left(Z>3 C_{p}\right)$

وبصورة عامة كلما انخفضت قيمة C باتجاه الصفر كلما زادت نسبة العيوب أو الوحدات غير المطابقة للمواصفات، الجدول (1) يبين العلاقة بين نسبة مقدرة العملية ونسبة الوحدات غير المطابقة للمواصفات. الجدول (1): نسب العيوب الدقابلة لبعض نسب مقدرة العملية C

\begin{tabular}{|c|c|c|}
\hline \multicolumn{2}{|c|}{ نسبة العيوب } & \multirow{2}{*}{$C_{p}$} \\
\hline (جزء من المليون) & $(\%)$ & \\
\hline 133,600 & 13.3600 & 0.50 \\
\hline 35,800 & 3.5800 & 0.70 \\
\hline 7,000 & 0.7000 & 0.90 \\
\hline 318.2 & 0.0967 & 1.10 \\
\hline 63.4 & 0.00962 & 1.30 \\
\hline 6.8 & 0.00267 & 1.50 \\
\hline 0.002 & 0.0000574 & 2.00 \\
\hline
\end{tabular}

إن قيمة Cساعد في فهم كفاءة العملية، فعلى سبيل المثال إذا كانت 1.33 > 1.30 للعناصر غير المطابقة للمواصفات التي تبلغ (63) جزءأ من المليون (parts per million) (ppm) فإن أداء

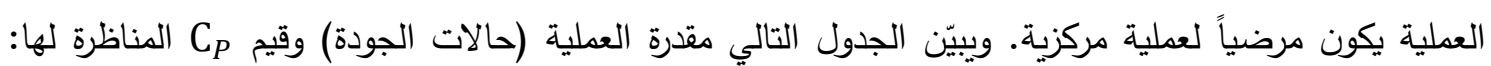
(Arcidiacono \& Nuzzi, 2017)

الجدول (2): مقدرة العملية وقيم C المناظرة لعملية مركزية

\begin{tabular}{|c|c|}
\hline مؤشر مقدرة العملية C & مقدرة العملية \\
\hline$C_{P} \geq 2.00$ & مدتازة جداً \\
\hline $1.67 \leq C_{P}<2.00$ & 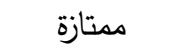 \\
\hline $1.33 \leq C_{P}<1.67$ & مقنعة \\
\hline $1.00 \leq C_{P}<1.33$ & لها مقدرة \\
\hline $0.67 \leq C_{P}<1.00$ & غير كافية \\
\hline$C_{P}<0.67$ & ليس لها مقدرة \\
\hline
\end{tabular}

وتعدُّ قيم C التي تبلغ 1.33 أو أعلى من معايير مقارنات الصناعة، هذا يعني أنَّ العمليّة متضمّنة في أربعة انحرافات معيارية لمواصفات العملية. 
Process Capability Index (PCI) مؤشر مقدرة العملية: 2.2

يوفر C معلومات فقط حول السعة النظرية للعملية؛ لأثّها لا تأخذ في الاعتبار مكان متوسط العملية نسبةً

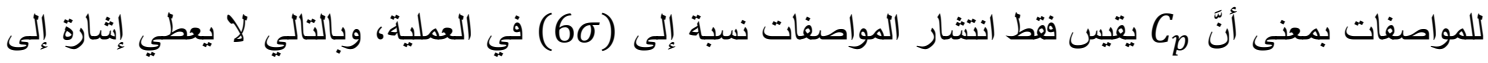
أداء العملية الفعلي.

يستخدم مؤشر مقدرة العملية C لربط اختلافات العملية من خلال إظهار كيفية توافق العملية مع

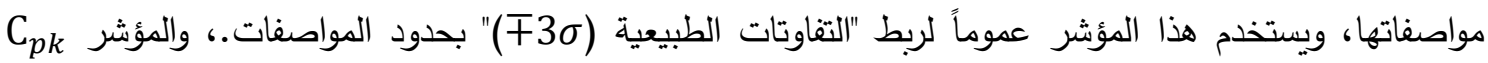
يصف بصورة جيدة تضمين العملية ضمن حدود المواصفات مع الإثارة إلى متوسط العملية؛ إذ: $C_{p k}=\min \left(C_{p k l}, C_{p k u}\right)$

$C_{p k u}=\frac{U S L-\mu}{3 \sigma}$ إذ إن Cمثل الحد الأعلى لـ

$C_{p k l}=\frac{\mu-L S L}{3 \sigma}$

المؤشر $C_{p k}$ يعطي معلومات حول المقدرة الحقيقية للعملية.

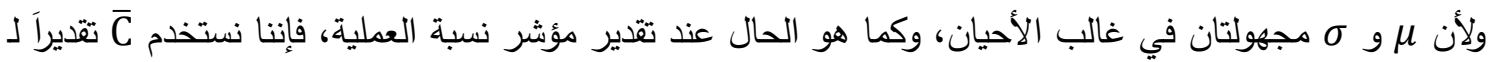
$C_{p k}=\min \left(\hat{C}_{p k l}, \hat{C}_{p k u}\right)$ إذ

$\hat{C}_{p k u}=\frac{U S L-\bar{X}}{3\left(\bar{R} / d_{2}\right)}$

$\hat{C}_{p k l}=\frac{\bar{X}-L S L}{3\left(\bar{R} / d_{2}\right)}$

3. مؤشرات أداء العملية في حالة المجتمعات غير الطبيعية:

\section{Process Capability Indices for Non-Normal Populations}

Xتفرض المعادلات المستخدمة لحساب جميع مؤشرات مقدرة العملية السابق ذكرها أن توزيع خاصية الجودة

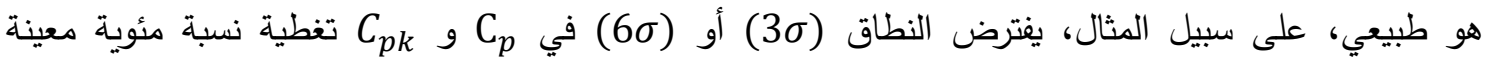
للخصائص تحت المنحنى الطبيعي (يغطي النطاق 6 ما ما مقداره 99.73\%). هذا الافتراض للطبيعية مهم؛ لأن

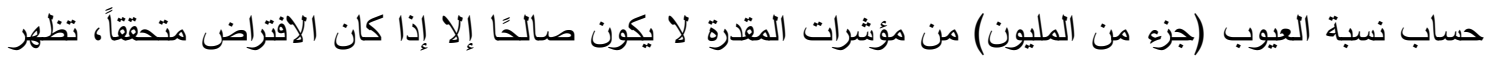
أدبيات البحث أن حساب PCI’s في ظل التوزيع غير الطبيعي للبيانات قد تمحور في مسارين رئيسين: (Korkusuz, 2011) PCI's وأدائها لمختلف التوزيعات المعروفة.

(2) بناء مؤشرات مقدرة جديدة و / أو تطوير أساليب جديدة مصممة خصيصاً للمخرجات غير الموزعة طبيعياً. وعلى الرغم من بذل الكثير من الجهود في هذه الدراسات، لا يوجد حتى الآن أي منهج قياسي معين أو مؤي مؤشر

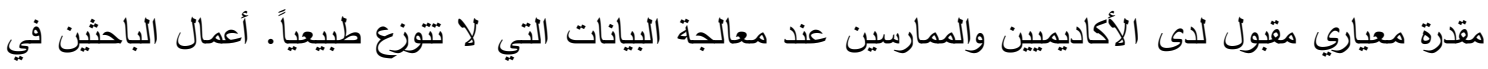
المسار الثاني صنفت إلى خمس مجموعات، وهي على النحو الآتي: 
Data Transformation Methods : 1.3 طرق تحويل البيانات

تهدف مناهج تحويل البيانات إلى تحويل بيانات العملية غير الطبيعية إلى بيانات عملية ذات توزيع طبيعي. اقترح العديد من الطرائق لتقريب البيانات الموزعة طبيعيا باستخدام الدالات الرياضية، من أشهر هذه الأساليب نظام تحويل جونسون Johnson الذي يعتمد على اشتقاق عزوم التوزيع، وتحويل القوى لـ بوكس-كوكس Box-Cox.

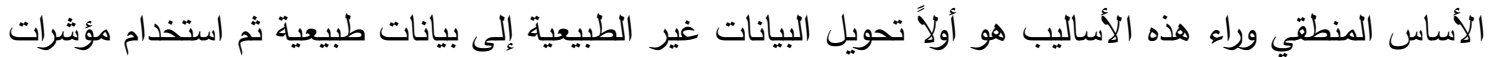
مقدرة العملية المعيارية التي تستند إلى افتراض الوضع الطبيعي للبيانات المحولة، ومع ذلك فإنَّ أساليب التحويل

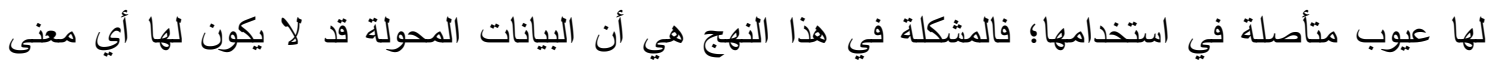
مادي، وقد لا يشعر المستخدمون بالارتياح مع البيانات المحولة. كما أنَّه أحياناً تفشل هذه التحويلات في ملاءمة التوزيع الطبيعي؛ ولأن تحويل Box-Cox هو الأكثر شيوعاً واستخداماً لذلك سيقتصر الحديث والتطبيق في الجانب العملي للبحث على هذا التحويل.

تحويل القوى لـ بوكس -كوكس: Box-Cox Power Transformation المبدأ الأساسي لهذه الطريقة يقوم على تحويل البيانات الأصلية التي لا تتبع التوزيع الطبيعي إلى بيانات ذات توزيع طبيعي وفق الصيغة الآتية:

$X^{(\lambda)}= \begin{cases}\frac{X^{\lambda}-1}{\lambda} & \text { if } \lambda \neq 0 \\ \operatorname{Ln} X & \text { if } \lambda=0\end{cases}$

إذ يختار أفضل قيمة لـ $\lambda$ بحيث يتحقق عندها أقل انحراف معياري للبيانات المحولة، وقد لوحظ أنَّ مدى قيم $\lambda$ المات

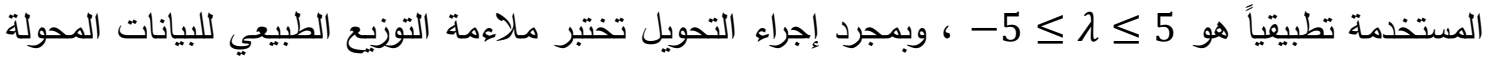
من إحدى اختبارات جودة مطابقة التوزيعات؛ إذ تنص فرضية العدم على ملاءمة التوزيع الطبيعي للبيانات في حين تثير الفرضية البديلة إلى خلاف ذلك.

2.3. لوحات السيطرة النوعية للتوزيعات غير الطبيعية:

\section{Quality Control Charts for Non-Normal Distributions}

بعض الأبحاث توجهت للاستقصاء في مقدرة العملية في حالة توزيعات أساسية معينة غير طبيعية، في هذه

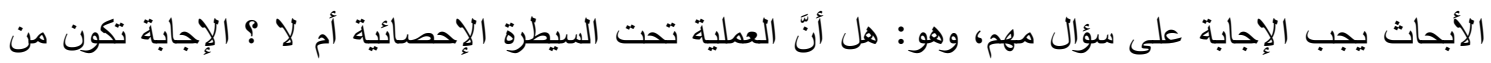

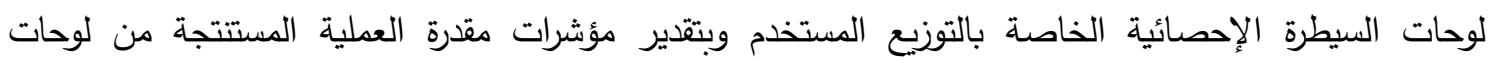
السيطرة هذه. هناك لوحات سيطرة طورت لكل من التوزيع اللوغاريتمي الطبيعي وتوزيع ويبل، وبعض الإتئ الباحثين اقترحوا استخدام لوحة متوسط المدى midrange ولوحة النسبة ratio من أجل تتبع الاتجاه المركزي والتشتت

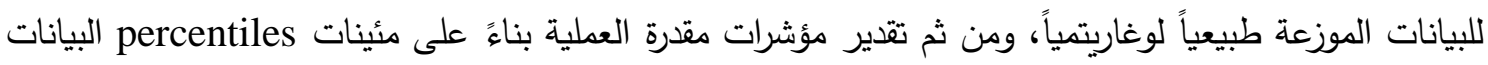

التجريبية. (Shore, 1998)

3.3. ملائمة التوزيعات للبيانات التجريبية: Distribution Fitting for Empirical Data تستخدم طرائق ملاءمة التوزيع بيانات العملية التجريبية التي يكون توزيعها غير معروف، وقد اقترح العديد من

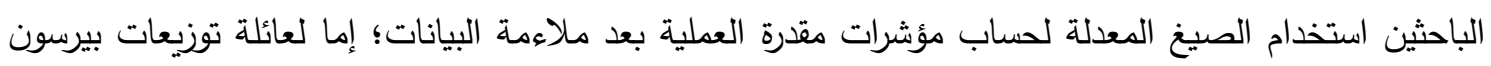


Pearson

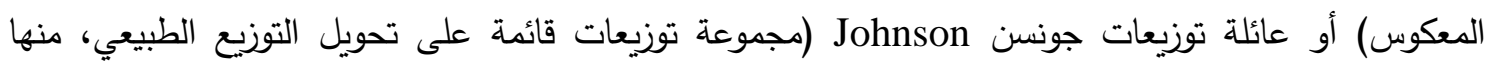
التوزيع الطبيعي اللوغاريتمي)، أو أي توزيع مناسب آخر استخدام الصيغ المعدلة لحساب مؤشرات مقدرة العملية.

(Chandra, 2001)

اقترح Clements الطريقة الآتية لمجموعة توزيعات بيرسون التي تعتمد على حساب المئينات Parga, (Kovarik \& Sarga, 2014) • اعتماداً على قيمة معامل تفلطح البيانات (Kurtosis) وقيمة معامل الالتواء للبيانات (Skeness)، ومن

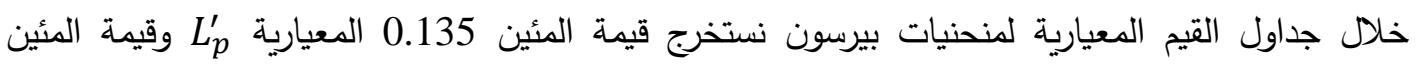

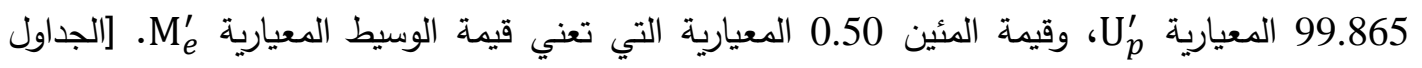
[توافرة في (Elisson, 2017)

$X_{0.00135}=\bar{X}-S * L_{P}^{\prime}$

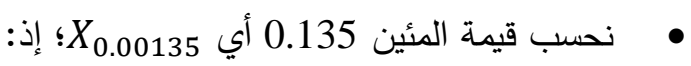

$X_{0.99865}=\bar{X}+S * U_{P}^{\prime}$ ونحسب قيمة المئين

$M e=X_{0.50}=\bar{X}+S * M_{e}^{\prime}$ ونحسب قيمة المئين 50 أي

$\hat{C}_{p}(C L)=\frac{U S L-L S L}{X_{0.99865}-X_{0.00135}}$

نحسب مؤشرات مقدرة العملية بالمعادلات الآتية:

$\hat{C}_{p l}(C L)=\frac{M e-L S L}{M e-X_{0.00135}}$

$\hat{C}_{p u}(C L)=\frac{U S L-M e}{X_{0.99865}-M e}$

$\hat{C}_{p k}(C L)=\min \left(\hat{C}_{p l}(C L), \hat{C}_{p u}(C L)\right)$

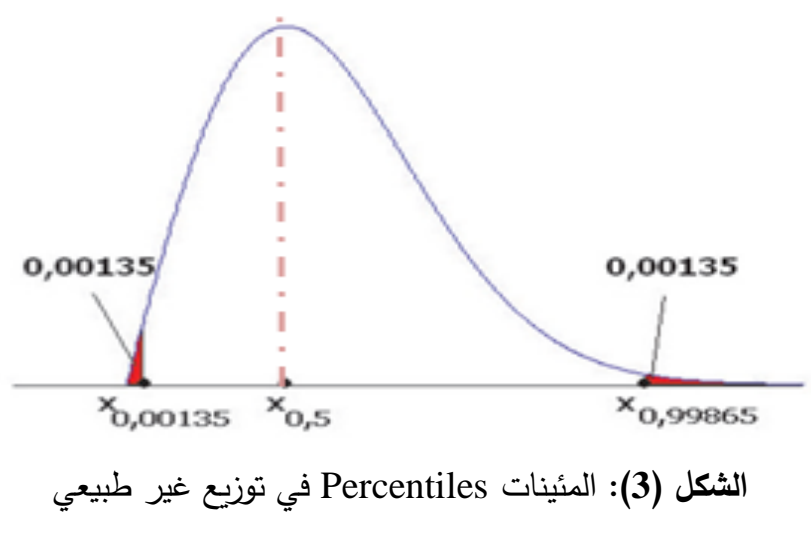

4.3. أساليب خالية من التوزيعات: Distribution-Free Approaches

تهدف هذه المناهج إما إلى تحديد فترات مواصفة خالية من التوزيع أو ضبط مؤشرات مقدرة العملية من خلال

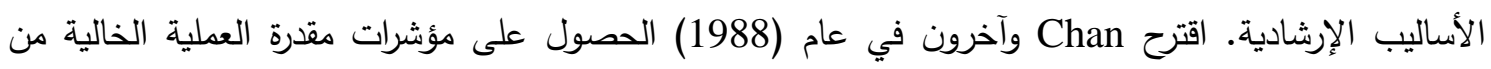

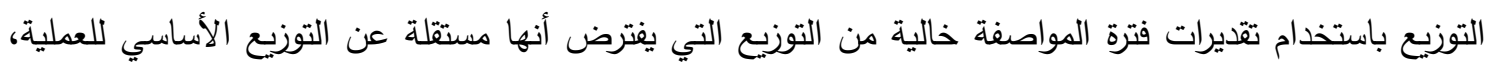


ومع ذلك فإن بناء فترات التفاوت أو السماح مشتقة من فترات التوزيع الطبيعي، وانتقد عدد من الباحثين هذا النهج؛ بسبب اعتماد نهج "خالٍٍ من التوزيع" على التوزيع الطبيعي. اقترحت طريقة التباين الموزون Weighted Variance بواسطة Choi و Bai في (1996)، جوهر طريقة التباين الموزون، ويعني تقسيم التوزيع غير الطبيعي الملتوي من متوسطه إلى توزيعين؛ وبذلك ينتج لدينا توزيعان جديدان بالمتوسط نفسه؛ ولكن بتباينات مختلفة لمجتمع ذي توزيع طبيعي بمتوسط بر وانحراف معياري $\sigma$ وسيكون

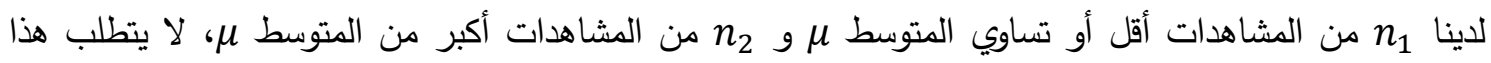

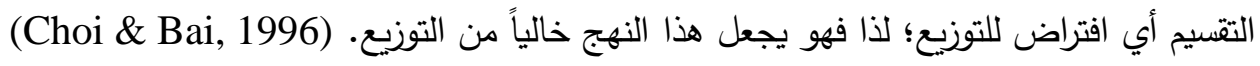
$\hat{\mu}=\bar{X}=\frac{1}{n} \sum_{i=1}^{n} X_{i}$ كما هو معلوم فإن $\mu$ تقدر حسب المعادلة الآتية: $\hat{\sigma}_{1}^{2}=S_{1}^{2}=\frac{2 \sum_{i=1}^{n_{1}}\left(X_{i}-\bar{X}\right)^{2}}{2 n_{1}-1}$ وبذلك ستكون معلمات التوزيع الأول هي ب و م والتي تقدر على النحو الآتي: $\hat{\sigma}_{2}^{2}=S_{2}^{2}=\frac{2 \sum_{i=1}^{n_{2}}\left(X_{i}-\bar{X}\right)^{2}}{2 n_{2}-1}$ ومعلمات التوزيع الثاني ستكون $\mu$ و $\hat{C}_{p}(W V)=\frac{U S L-L S L}{3\left(S_{1}+S_{2}\right)}$ نحسب مؤشرات مقدرة العملية بالمعادلات الآتية:

$\hat{C}_{p l}(W V)=\frac{\bar{X}-L S L}{3 S_{1}}$

$\hat{C}_{p u}(W V)=\frac{U S L-\bar{X}}{3 S_{2}}$

$\hat{C}_{p k}(W V)=\min \left(\hat{C}_{p l}(W V), \hat{C}_{p u}(W V)\right)$

5.3. مؤشرات جليدة لمقدرة البيانات غير الطبيعية: New PCI’s for Non-Normal Data

بذل قدر كبير من العمل لتطوير مؤشرات مقدرة العملية التي ستكون حصينة ضد الخاصية غير الطبيعية

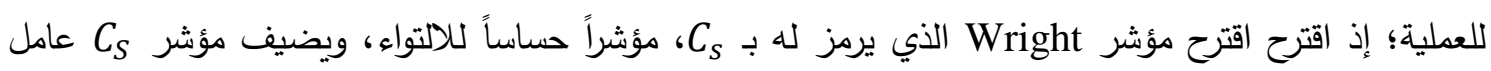

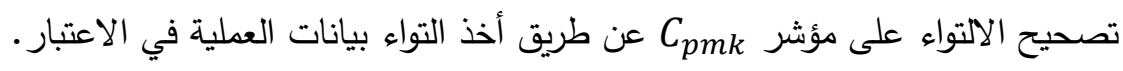
واستتنج مؤشر مرن لمقدرة العملية وهو C C C ، بوساطة Johnson وآخرون في عام (1994). يعتمد هذا المؤشر على مؤشر Com ويفترض أنه مرن؛ لأن عدم التماثل في العملية غير الطبيعية يؤخذ مع الفرق في التشتت بين

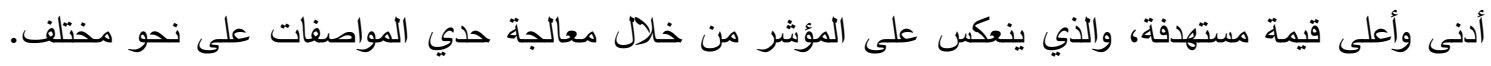

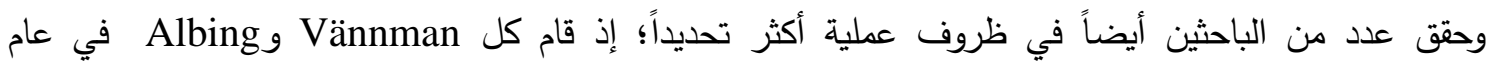

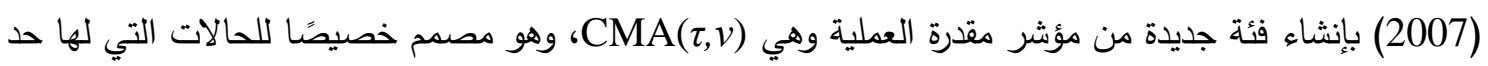

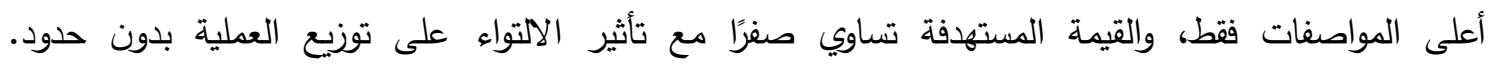

(Korkusuz, 2011) 
أخذت البيانات من المصدر (سمير علي و طارق محمد، 2.13)؛ إذ يمثل المتغير الرئيس لبيانات البحث القطر الداخلي لكأس الزنك في البطارية الجافة من النوع الكبير (مليمتر mm) في أحد مصانع إنتاج البطاريات الجافة، وحدد المصمم قطر كأس الزنك في البطارية الجافة بـ (27.9 F 0.3 mm)، وهذا يعني أن الحد الأعلى للمواصفات المطلوبة سيكون USL = 28.2 والحد الأدنى للمواصفات سيكون الهدف 29.7 .Target سحبت عينة عشوائية بحجم ( 5فردات) يومياً ولمدة (25 يوماً) أي إنَّ (n=5 , k=25) وسجلت البيانات في

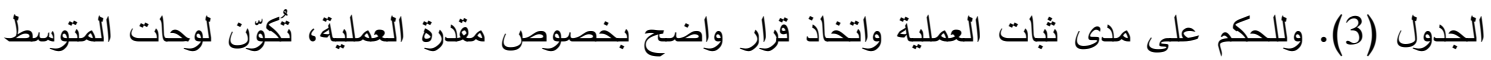

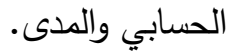
الجدول (3): المتوسطات الحسابية والمديات لعينات بيانات البحث

\begin{tabular}{|c|c|c|c|c|c|c|c|}
\hline Sample & $X_{1}$ & $X_{2}$ & $X_{3}$ & $X_{4}$ & $X_{5}$ & $\bar{X}_{j}$ & $R_{j}$ \\
\hline \hline 1 & 27.60 & 27.84 & 27.98 & 27.84 & 27.60 & 27.77 & 0.38 \\
\hline 2 & 27.96 & 27.84 & 27.70 & 27.62 & 27.76 & 27.78 & 0.34 \\
\hline 3 & 27.65 & 27.71 & 27.70 & 27.85 & 27.73 & 27.73 & 0.20 \\
\hline 4 & 28.04 & 27.65 & 28.05 & 27.96 & 27.71 & 27.88 & 0.40 \\
\hline 5 & 27.64 & 27.65 & 28.19 & 27.95 & 27.85 & 27.86 & 0.55 \\
\hline 6 & 28.20 & 27.70 & 27.65 & 28.11 & 27.65 & 27.86 & 0.55 \\
\hline 7 & 27.75 & 27.78 & 27.63 & 27.68 & 27.70 & 27.71 & 0.15 \\
\hline 8 & 28.09 & 28.01 & 27.96 & 27.40 & 27.65 & 27.82 & 0.69 \\
\hline 9 & 27.74 & 28.05 & 27.60 & 27.95 & 27.89 & 27.85 & 0.45 \\
\hline 10 & 28.08 & 28.00 & 27.84 & 28.04 & 28.06 & 28.00 & 0.24 \\
\hline 11 & 27.99 & 27.87 & 27.92 & 27.94 & 28.03 & 27.95 & 0.16 \\
\hline 12 & 27.72 & 27.82 & 27.91 & 27.92 & 27.84 & 27.84 & 0.20 \\
\hline 13 & 27.85 & 27.88 & 27.88 & 27.84 & 27.83 & 27.86 & 0.05 \\
\hline 14 & 27.73 & 27.81 & 27.84 & 27.81 & 27.75 & 27.79 & 0.11 \\
\hline 15 & 27.69 & 27.58 & 27.64 & 28.01 & 27.98 & 27.78 & 0.43 \\
\hline 16 & 27.90 & 28.16 & 27.95 & 27.85 & 28.28 & 28.03 & 0.43 \\
\hline 17 & 27.88 & 27.75 & 27.83 & 27.92 & 27.76 & 27.83 & 0.17 \\
\hline 18 & 27.75 & 27.71 & 27.87 & 28.00 & 27.80 & 27.83 & 0.29 \\
\hline 19 & 27.86 & 27.78 & 27.66 & 27.76 & 28.05 & 27.82 & 0.39 \\
\hline 20 & 27.78 & 27.77 & 27.62 & 27.78 & 28.19 & 27.83 & 0.57 \\
\hline 21 & 27.94 & 28.25 & 27.58 & 27.71 & 27.75 & 27.85 & 0.67 \\
\hline 22 & 27.87 & 27.72 & 27.60 & 28.02 & 27.84 & 27.81 & 0.42 \\
\hline 23 & 27.97 & 27.88 & 27.72 & 27.87 & 27.90 & 27.87 & 0.25 \\
\hline 24 & 27.88 & 28.18 & 27.90 & 27.56 & 27.86 & 27.88 & 0.62 \\
\hline 25 & 27.76 & 27.92 & 27.89 & 27.83 & 27.95 & 27.87 & 0.19 \\
\hline
\end{tabular}


أولاً: لوحة المتوسط الحسابي:

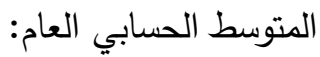

$\overline{\bar{X}}=\frac{1}{k} \sum_{j=1}^{k} \bar{X}_{j}=\frac{696.07}{25}=27.843$

المتوسط الحسابي للمديات:

$\bar{R}=\frac{1}{k} \sum_{j=1}^{k} R_{j}=\frac{8.90}{25}=0.356$

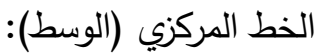

$C L=\overline{\bar{X}}=27.843$

حد السيطرة الأعلى للمتوسط:

$U C L=\overline{\bar{X}}+A_{2} \bar{R}=27.843+0.577 \times 0.356=28.048$

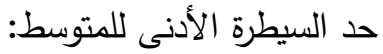

$L C L=\overline{\bar{X}}-A_{2} \bar{R}=27.843-0.577 \times 0.356=27.638$

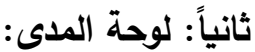

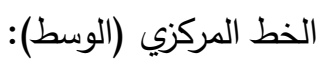

$C L=\bar{R}=0.356$

حد السيطرة الأعلى للمدى: - مل

$U C L=D_{4} \bar{R}=2.115 \times 0.356=0.753$

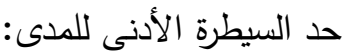

$L C L=D_{3} \bar{R}=0 \times 0.24=0$

إذ تستخرج قيم الثوابت للمتغيرات (Montgomery, 2013).

اعتماداً على هذه النتائج وباستخدام البرمجية Minitab-17 كانت المخرجات كما في الثكل (4)؛ إذ يتبين من الثكل أن جميع متوسطات العينات واقعة ضمن حدي السيطرة الأعلى والأدنى للمتوسط، وكذلك الحال بالنسبة لجميع مديات العينات فهي واقعة بين حدي السيطرة للمدى، نستتتج من ذلك أنَّ عملية صنع القطر الداخلي لكأس الزنك في البطارية الجافة مستقرة وخاضعة للسيطرة النوعية، وأنَّه يمكن حساب مؤشرات مقدرة العملية. 


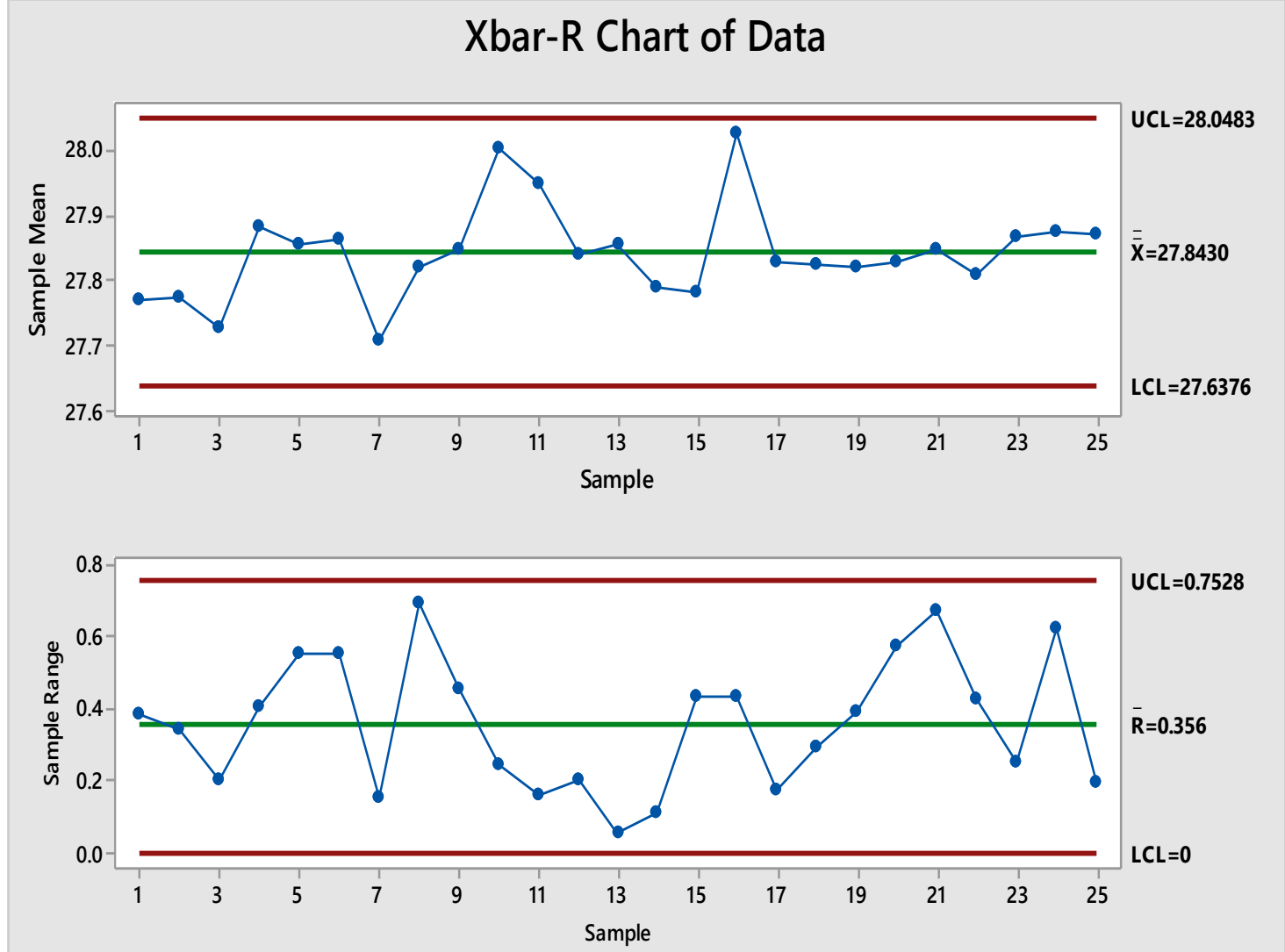

الثكل (4): لوحات السيطرة النوعية للمتوسط والمدى لبيانات البحث

5. تكوين المدرج التكراري وإختبار التوزيع الطبيعي للبيانات:

لغرض اختبار مدى ملاعمة التوزيع الطبيعي لبيانات البحث سيكوّن أولاً الجدول التكراري لبيانات الجدول

(3)؛ وذلك باختيار عدد ملائم من الفئات (7-12 فئة) اعتماداً على حجم العينة الكلي للبيانات (N=nk=125). (عطا، 2012)

الجدول (4): جدول التوزيع التكراري لبيانات الجدول (3)

\begin{tabular}{|c|c||}
\hline Class & Frequency \\
\hline $27.40-27.52$ & 1 \\
\hline $27.52-27.64$ & 10 \\
\hline $27.64-27.76$ & 30 \\
\hline $27.76-27.88$ & 34 \\
\hline $27.88-28.00$ & 28 \\
\hline $28.00-28.12$ & 15 \\
\hline $28.12-28.24$ & 5 \\
\hline $28.24-28.36$ & 2 \\
\hline \hline Total & 125 \\
\hline
\end{tabular}


تستخدم الطرائق البيانية المتضمنة المدرج التكراري ورسم احتمالات التوزيع الطبيعي؛ لغرض التحقق من التوزيع الطبيعي للبيانات، ويتضح من المدرج التكراري في الثكل (5) أنَّ بيانات البحث لاتئ لتبع التوزيع الطبيعي.

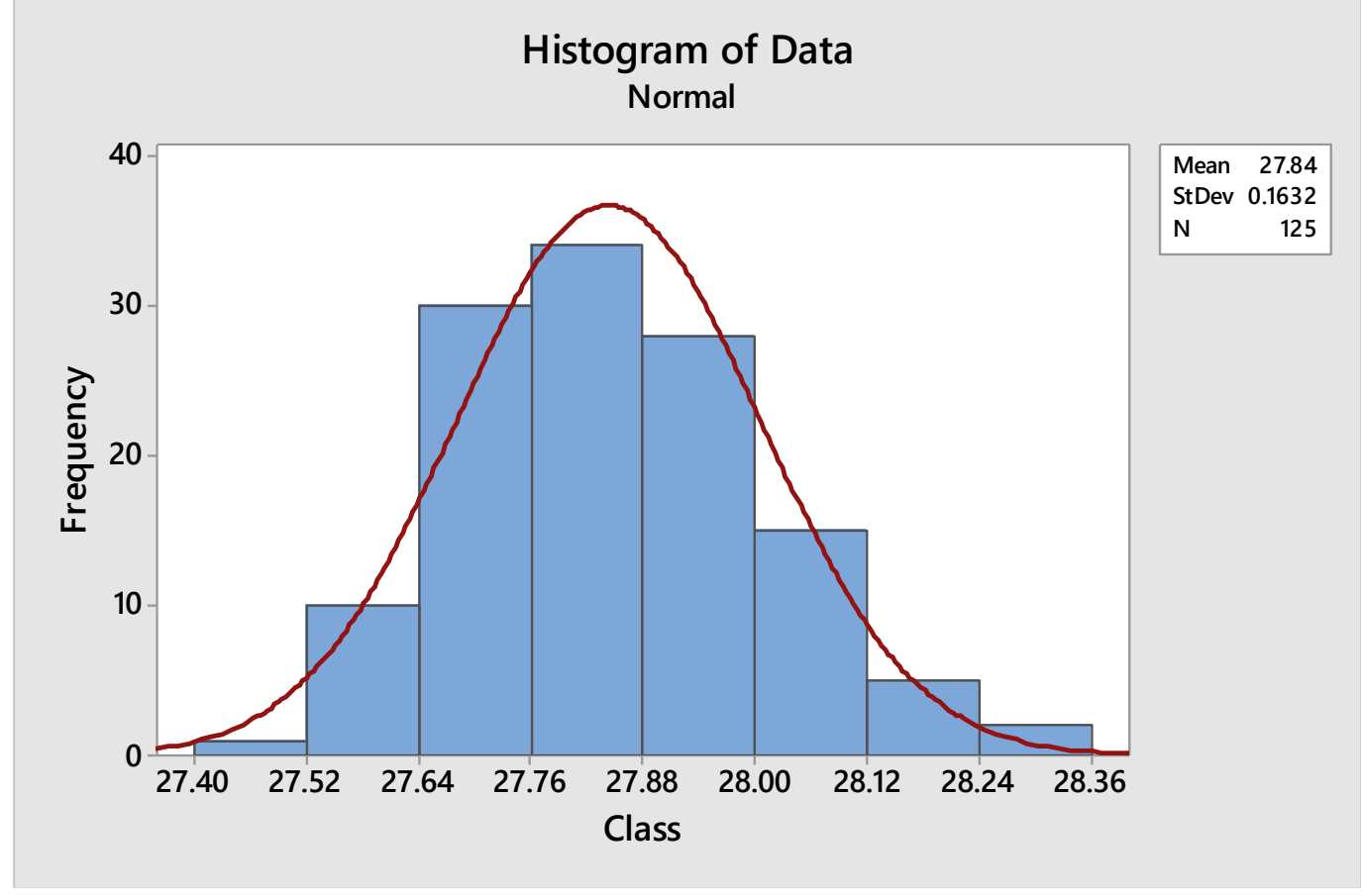

$$
\text { الثكل (5): الدرج التكراري لبيانات البحث }
$$

وكذلك برسم احتمالات التوزيع الطبيعي Normal Probability Plot للبيانات يتضح عدم وقوع القير على الخط المستقيم كما أنَّ البعض منها واقع خارج فترة الثقة للتوزيع الطبيعي البالغة (95\%).

\section{Probability Plot for Data}

Normal $-95 \% \mathrm{Cl}$

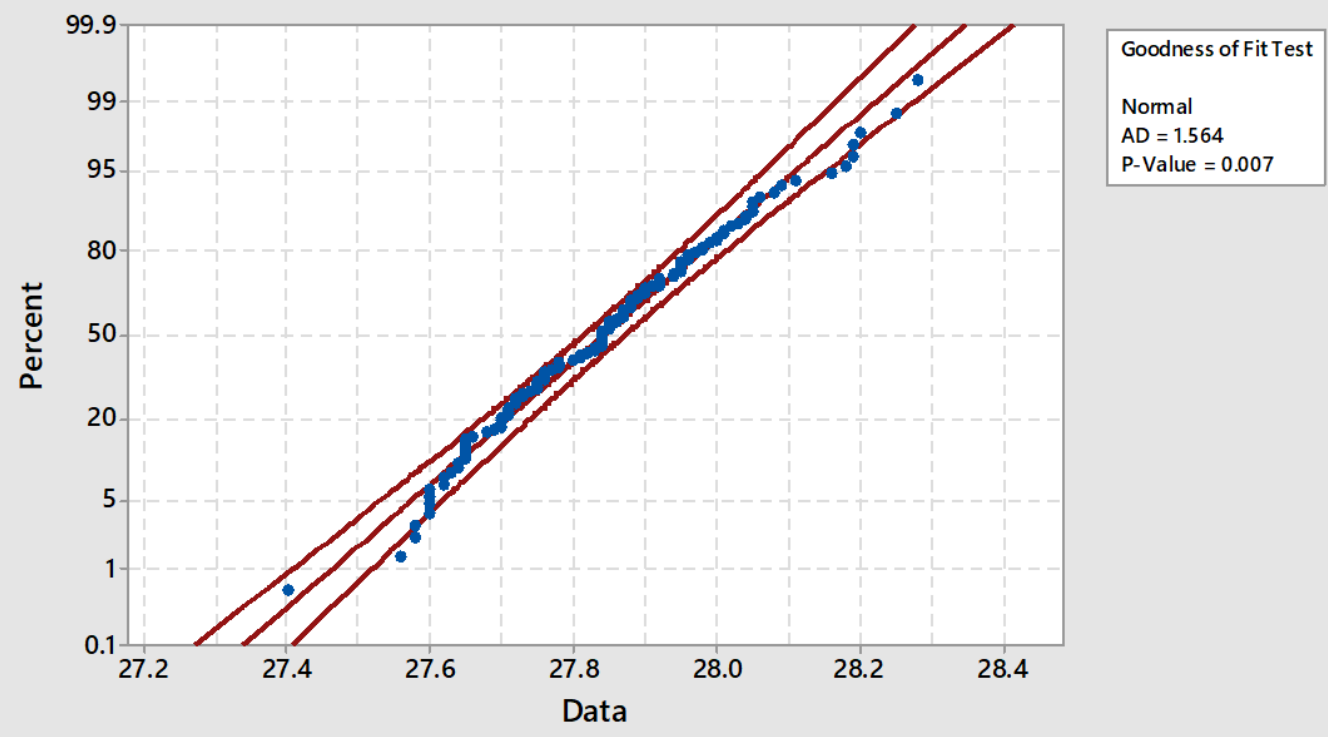

$$
\text { الثكل (6): رسم احتمالات التوزيع الطبيعي لبيانات البحث }
$$


Minitab-17 لبيان مدى جودة مطابقة التوزيع الطبيعي للبيانات، واعتماداً على نتائج البرمجية AD واستخدم أظهرت نتائج الاختبار أن قيمة الاحتمال P-Value=0.007 للاختبار التي هي أقل من (5\%) مما يعني رفض فرضية العدم وقبول الفرضية البديلة، وبذلك نستنتج أن القطر الداخلي لكأس الزنك في البطارية الجافة لا يتبع التوزيع الطبيعي.

\section{6. تقدير مؤشرات مقدرة العملية:}

سبق وأن أوضحنا أن بيانات البحث لا تتبع التوزيع الطبيعي، بالتالي فإنه لا يمكن الحديث عن مقدرة العملية

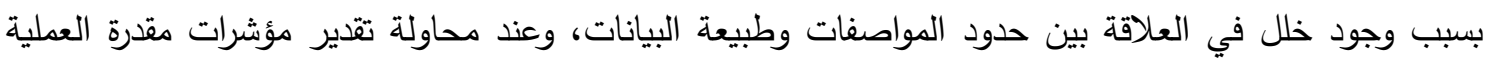
لبيانات البحث باستخدام المعادلات المتتاولة سابقاً تم الحصول على النتائج الآتية: $\hat{C}_{p}=0.61, \hat{C}_{p l}=0.49, \hat{C}_{p u}=0.73, \hat{C}_{p k}=\min (0.49,0.73)=0.49$

ورسمت البيانات مع حدود المواصفات على النحو الآتي:

\section{Process Capability Report for Data}

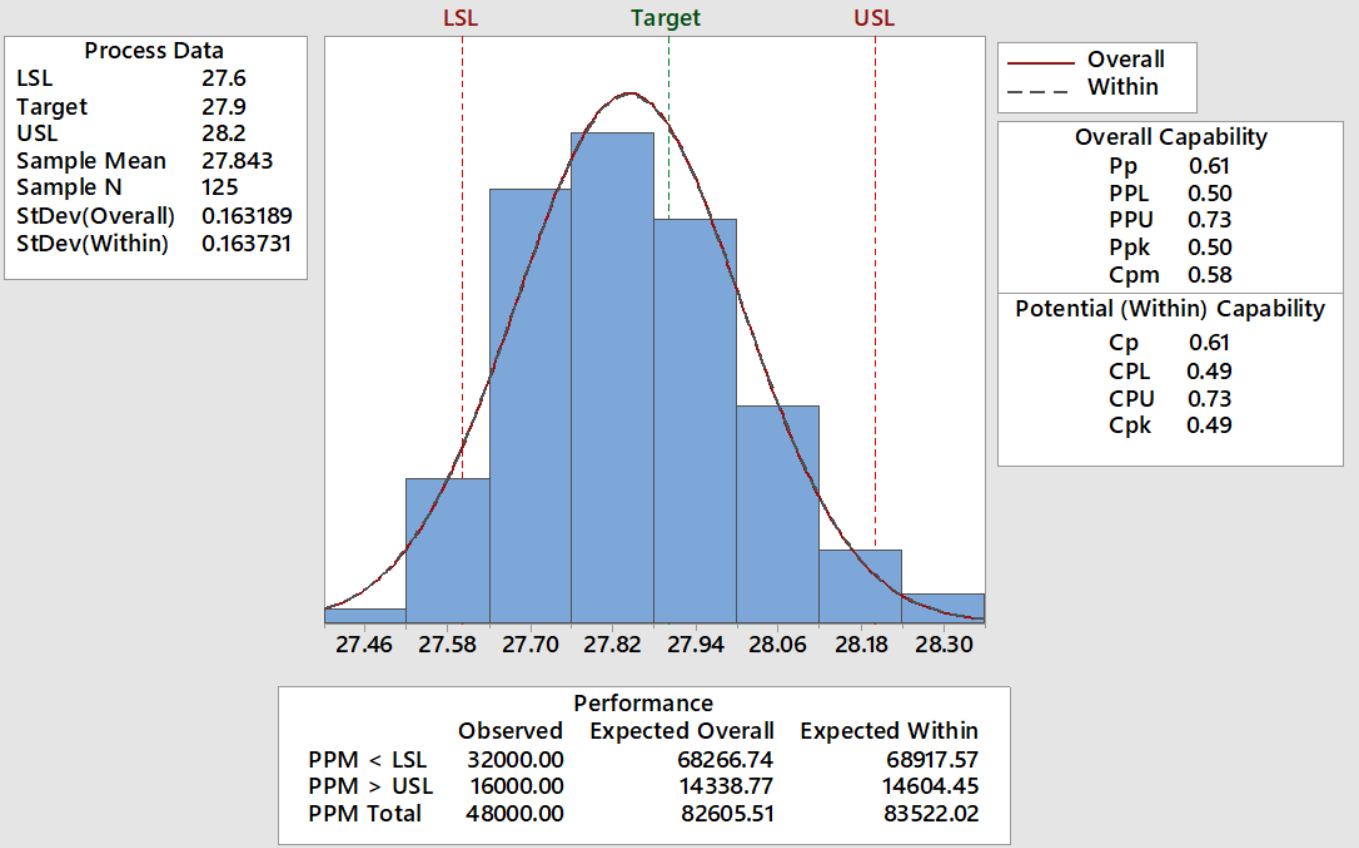

الثكل (7): نتائج مؤشرات مقدرة العملية لبيانات البحث

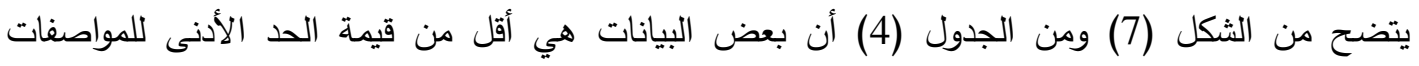
(LSL)

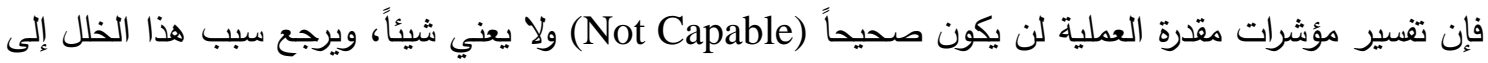

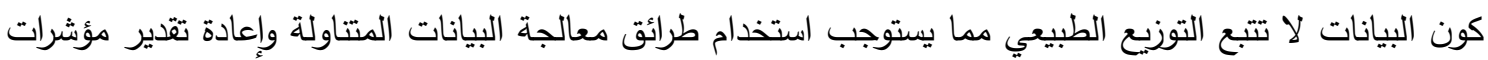
العملية بصورة صحيحة.

1.6. بريقة التحويل القوى لـ Cox-Box

المبدأ الأساسي لهذه الطريقة يقوم على تحويل بيانات البحث الأصلية التي لا تتبع التوزيع الطبيعي وفق

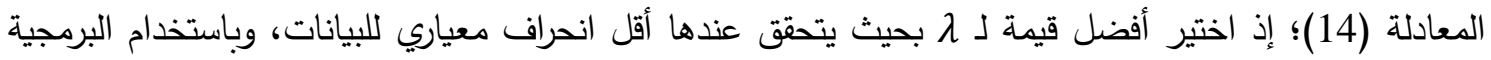


Minitab-17 تُوصِل إلى أنَّ أفضل قيمة لـ ג هي (5.00-) التي تقابل عندها أقل قيمة انحراف معياري للبيانات، وهذا يعني أنَّ تحويل Box-Cox المستخدم للبيانات هو: $X^{(\lambda)}=\frac{X^{\lambda}-1}{\lambda}=\frac{1}{5}\left(1-\frac{1}{X^{5}}\right)$

وللتحقق من أنَّ البيانات تتوزع توزيعاً طبيعياً بعد عميلة التحويل تمت إعادة اختبار AD؛ إذ كانت نتائج الاختبار

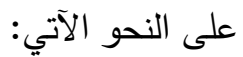
أظهرت نتائج الاختبار أنَّ قيمة الاحتمال P-Value=0.447 للاختبار التي هي أكبر من (5\%) ممّا يعني أن عملية تحويل البيانات باستخدام طريقة Box-Cox قد نجحت في جعل البيانات تتبع التوزيع الطبيعي وهذا ما يؤكده الثكل (8).

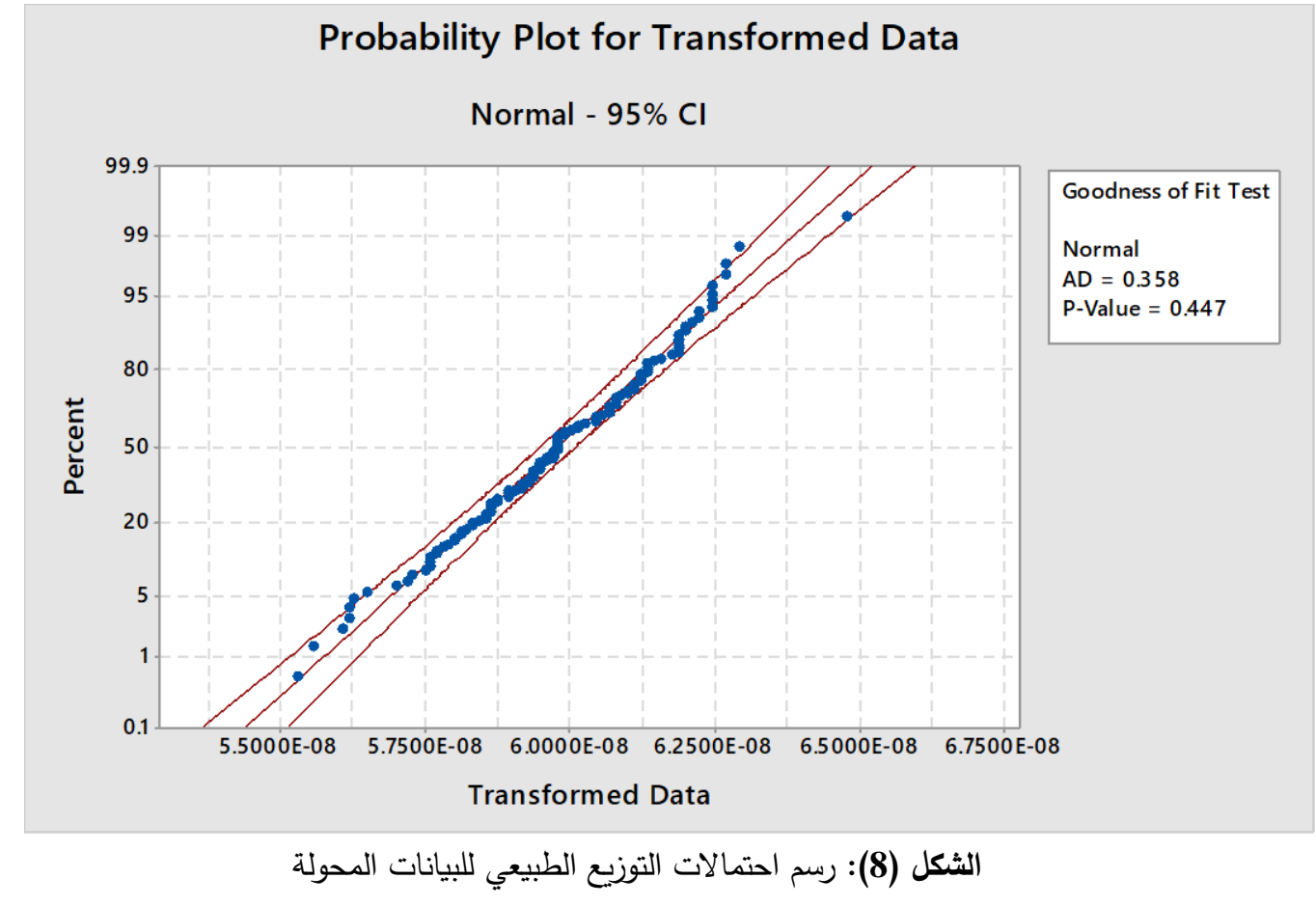

ورسمت البيانات المحولة مع حدود المواصفات كما في الثكل (9). ويتضح من الثكل أنَّ البيانات المحولة لا لاتهات زالت تعاني من خلل في تفسير مؤشرات مقدرة العملية بسبب عدم وقوع كافة البيانات المحولة بين حدي المواصفات الأدنى والأعلى. وقدرت مؤشرات مقدرة العملية للبيانات المحولة والحصول على النتائج الآتية: $\hat{C}_{p}(B C)=0.61, \hat{C}_{p l}(B C)=0.50, \hat{C}_{p u}(B C)=0.71$ $\hat{C}_{p k}(B C)=\min (0.50,0.71)=0.50$ 


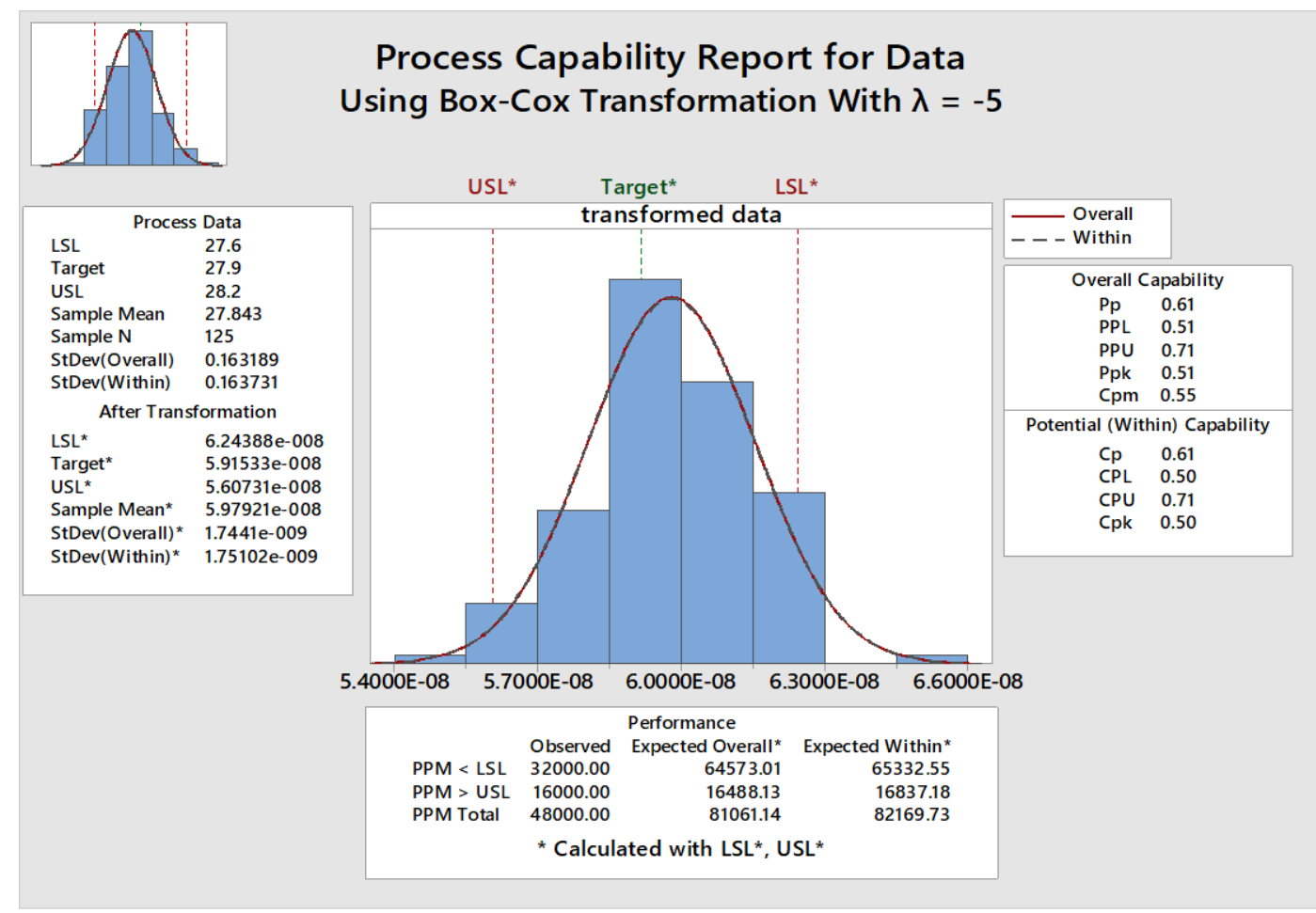

الثكل (9): نتائج مؤشرات مقدرة العملية للبيانات المحولة

2.6 - 2.6 - طريقة التباين الموزون:

أدناه خطوات تطبيق طريقة التباين الموزون على بيانات البحث:

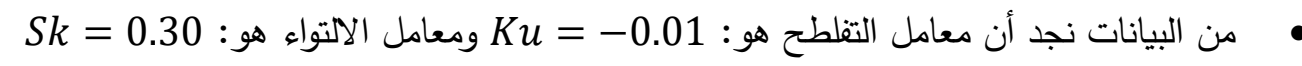

• نحسب مؤشرات مقدرة العملية بالاعتماد على المعادلات (30)، (31) و (32) وعلى النحو الآتي:

$\hat{C}_{p}(W V)=\frac{28.2-27.6}{3(0.1533+0.1733)}=\frac{0.6}{0.9798}=0.61$

$\hat{C}_{p l}(W V)=\frac{27.843-27.6}{3 * 0.1533}=\frac{0.243}{0.4599}=0.53$

$\hat{C}_{p u}(W V)=\frac{28.2-27.843}{3 * 0.1733}=\frac{0.357}{0.5199}=0.69$

$\hat{C}_{p k}(W V)=\min (0.53,0.69)=0.53$

:Clements 3.6 طريقة

أدناه خطوات تطبيق Clements على بيانات البحث:

اعتماداً على قيمة معامل التفلطح (Ku=-0.01) وقيمة معامل الالتواء الموجبة (Sk=0.30)،

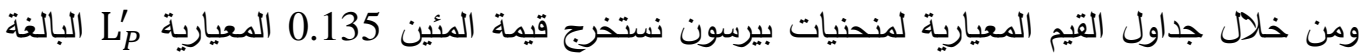

البالغة $L_{P}^{\prime}=2.329$ وقيمة المئين 99.865 المعيارية $U_{P}^{\prime}=3.133$ وكذلك قيمة الوسيط

$$
\text { المعياري } M_{e}^{\prime}=0.058 \text { البالغة }
$$

نحسب قيمة المئين 0.135 بالاعتماد على المعادلة (15):

$X_{0.00135}=27.843-0.163 * 2.329=27.463$

ونحسب قيمة المئين 99.865 بالاعتماد على المعادلة (16): 
$X_{0.99865}=27.843+0.163 * 3.133=28.354$

ونحسب قيمة الوسيط بالاعتماد على المعادلة (17):

$M e=X_{0.50}=27.843+0.163 * 0.058=27.852$

نحسب مؤشرات مقدرة العملية بالاعتماد على المعادلات (18)، (19) و (20):

$\hat{C}_{p}(C L)=\frac{28.2-27.6}{28.354-27.463}=\frac{0.6}{0.891}=0.67$

$\hat{C}_{p l}(C L)=\frac{27.852-27.6}{27.852-27.463}=\frac{0.252}{0.389}=0.65$

$\hat{C}_{p u}(C L)=\frac{28.2-27.852}{28.354-27.852}=\frac{0.348}{0.502}=0.69$

$\hat{C}_{p k}(C L)=\min (0.65,0.69)=0.65$

4.6. ملاءمة توزيعات أخرى لبيانات البحث:

استخدمت وظيفة التعرف على توزيع البيانات في البرمجية الإحصائية Minitab-17 لرسم الاحتمالات

ومقارنة نتائج اختبار جودة تمثيل هذه التوزيعات لبيانات البحث، واستخدمت سبعة توزيعات شائعة والمقارنة بينها اعتماداً على اختبار AD؛ إذ كانت النتائج كما في الجدول الآتي: الجدول (5): مقارنة بين التوزيعات الاحتمالية باستخدام نتائج رسم الاحتمالات

\begin{tabular}{||l||c|c|}
\hline \multicolumn{1}{|c||}{ Distribution Type } & AD Value & P-Value \\
\hline \hline Lognormal & $\mathbf{0 . 4 2 7}$ & $\mathbf{0 . 3 0 8}$ \\
\hline Gamma & 0.435 & $>0.250$ \\
\hline Exponential & 56.687 & $<0.003$ \\
\hline Smallest Extreme Value & 2.754 & $<0.010$ \\
\hline Weibull & 2.687 & $<0.010$ \\
\hline Largest Extreme Value & 0.792 & 0.040 \\
\hline Logistic & 0.465 & 0.206 \\
\hline
\end{tabular}

يلاحظ من نتائج الجدول السابق واعتماداً على القيم الاحتمالية لاختبار AD التي هي أكبر من (5\%) أنَّ كلاً من

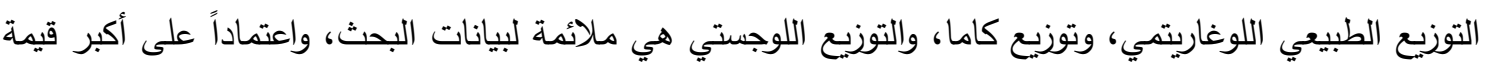

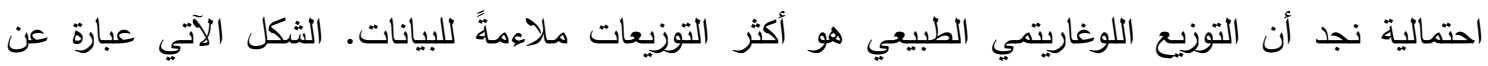
مخرجات Minitab-17 التي تتوضح رسم الاحتمالات للتوزيعات المتناولة. 

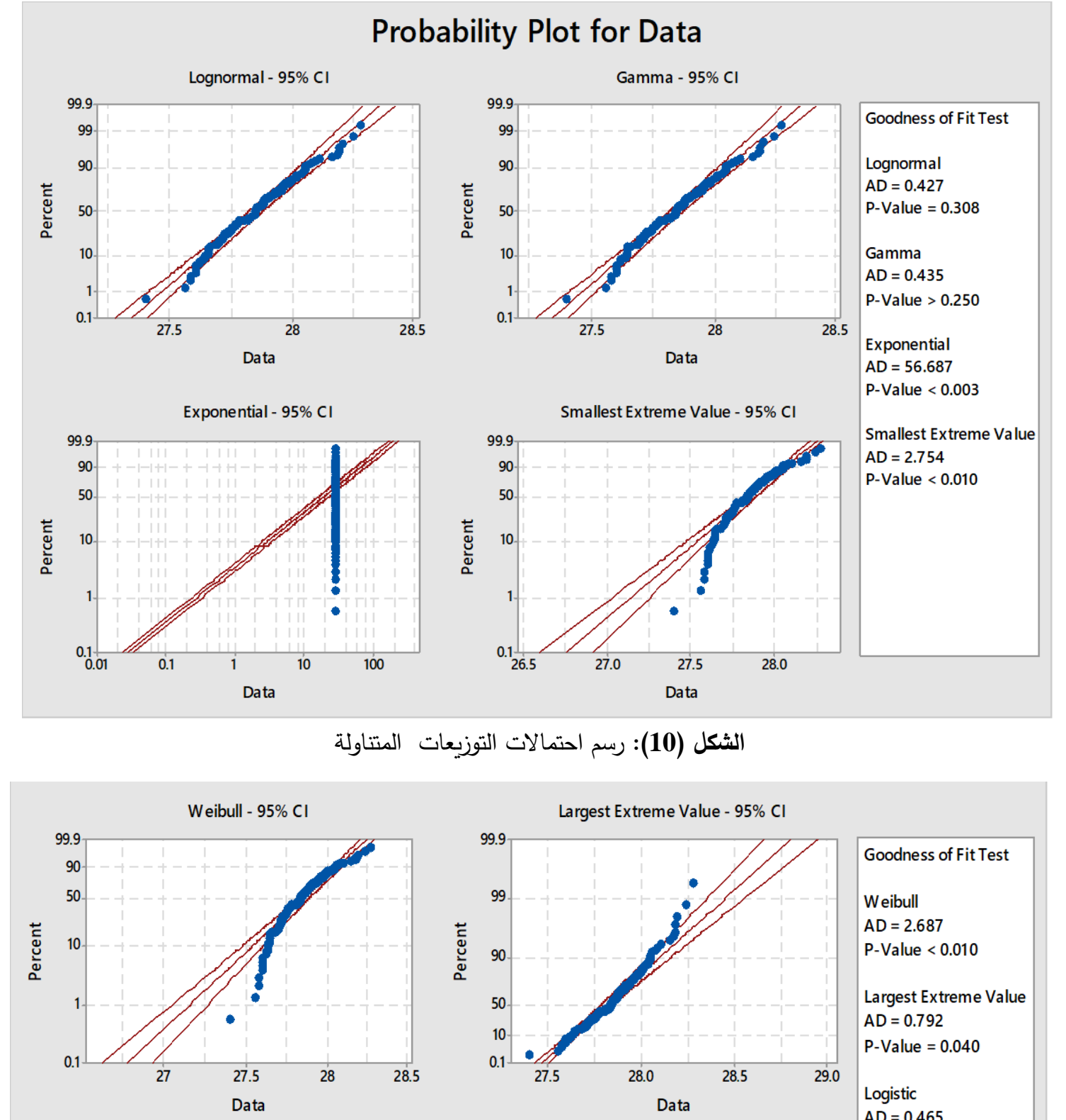

Goodness of Fit Test

Weibull

$A D=2.687$

P-Value $<0.010$

Largest Extreme Value

$A D=0.792$

$\mathrm{P}$-Value $=0.040$

Logistic

$\mathrm{AD}=0.465$

$\mathrm{P}$-Value $=0.206$

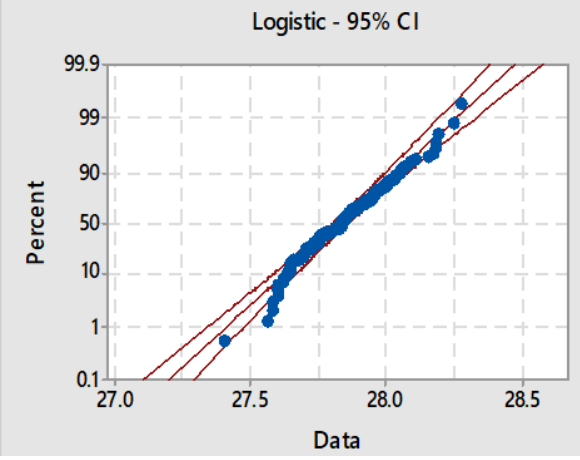

الثكل أدناه يبين نتائج ملاعمة التوزيع اللوغاريتمي الطبيعي لبيانات البحث مع مؤشرات مقدرة العملية. 


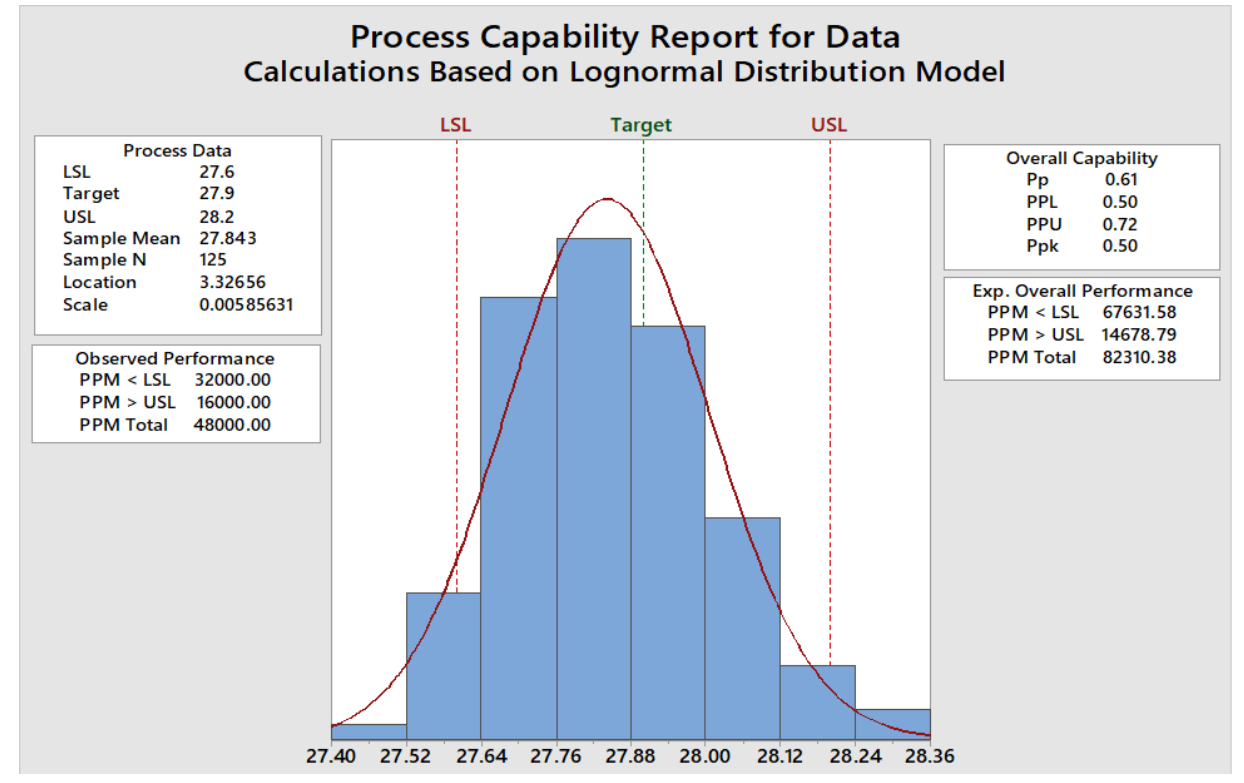

الثكل (11): نتائج مؤشرات مقدرة العملية للتوزيع الطبيعي اللوغاريتمي لبيانات البحث

من الثكل (11) فإن مؤشرات مقدرة العملية المبنية على التوزيح الطبيعي اللوغاريتمي هي: $\hat{C}_{p}(L N)=0.61, \hat{C}_{p l}(L N)=0.50, \hat{C}_{p u}(L N)=0.72$

$\hat{C}_{p k}(L N)=\min (0.50,0.72)=0.50$

7. مقارنة نتائج مؤشرات مقدرة العملية للطرائق المختلفة:

الجدول التالي يعرض نتائج تقدير مؤشرات مقدرة العملية بالطرائق المختلفة المتتاولة في هذا البحث.

الجدول (6): نتائج مؤشرات مقدرة العملية بالطريقة التقليدية والطرائق غير الطبيعية

\begin{tabular}{|c||c|c|c|c|c||}
\hline PCI's & $\begin{array}{c}\text { Classical method } \\
\text { (Normality } \\
\text { Assumption) }\end{array}$ & $\begin{array}{c}\text { Box-Cox } \\
\text { Method }\end{array}$ & $\begin{array}{c}\text { Clements } \\
\text { Method }\end{array}$ & $\begin{array}{c}\text { Weighted } \\
\text { Variance } \\
\text { Method }\end{array}$ & $\begin{array}{c}\text { Lognormal } \\
\text { Model }\end{array}$ \\
\hline \hline$\hat{C}_{p}$ & 0.61 & 0.61 & $\mathbf{0 . 6 7}$ & 0.61 & 0.61 \\
\hline$\hat{C}_{p l}$ & 0.49 & 0.50 & $\mathbf{0 . 6 5}$ & 0.53 & 0.50 \\
\hline$\hat{C}_{p u}$ & 0.73 & 0.71 & $\mathbf{0 . 6 9}$ & 0.69 & 0.72 \\
\hline$\hat{C}_{p k}$ & 0.49 & 0.50 & $\mathbf{0 . 6 5}$ & 0.53 & 0.50 \\
\hline
\end{tabular}

يلاحظ من نتائج الجدول السابق أن نتائج مؤشرات مقدرة العملية وبجميع الطرائق كانت أقل من الواحد الصحيح مما يثير ذلك إلى أنَّ عملية تصنيع القطر الداخلي لكأس الزنك في البطارية الجافة بوضعها الحالي ليس لها مقدرة، وأنَّا لا تلبي المواصفات المطلوبة بنسبة (100\%) مما يستوجب العمل الجدي بخصوص بعد مخرجات العملية الخارجة عن المواصفات، عموماً ومع ذلك، يمكن القول: إن نتائج طريقة Clements هي الأفضل مقارنةً بالطرائق الأخرى، ويتضح من ذلك أنَّ مقدرة العملية باستخدام طريقة Clements تستطيع تحقيق نسبة (65\%) من المواصفات المطلوبة. 8

ا. اعتماداً على الثكل (4) يتضح أنَّ عملية صنع القطر الداخلي لكأس الزنك في البطارية الجافة هي مستقرة وخاضعة للسيطرة النوعية. 
ץ. أظهرت نتيجة اختبار Anderson-Darling من خلال الثكلين (5) و (6) أنَّ بيانات القطر الداخلي لكأس

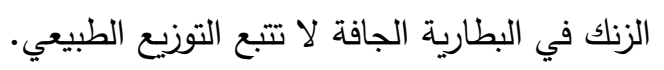

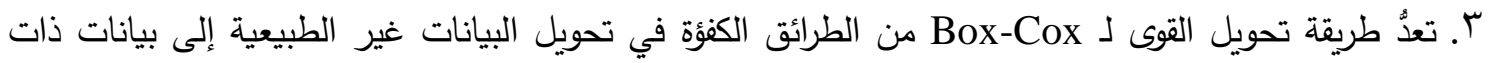
توزيع طبيعي، فمن خلال الثكلين (8) و (9) وباستخدام تحويل Box-Cox وعند معامل التحويل 5 -

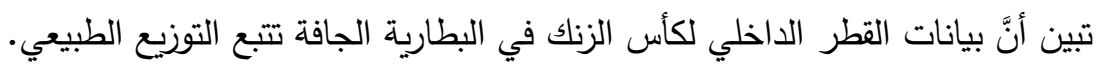
ع. من خلال نتائج جودة المطابقة في الجدول (5) وكذلك من خلال رسم احتمالات التوزيعات في الثكل (10) تبين أن هناك عدة توزيعات احتمالية متصلة تلائم بيانات القطر الداخلي لكأس الزنكك في البطارية الجافة وهي:

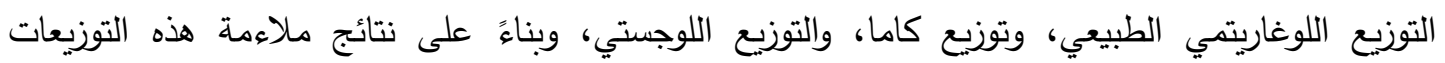
للبيانات، أتضح أن التوزيع اللوغاريتمي الطبيعي هو أفضل التوزيعات ملاءمةً لبيانات البحث.

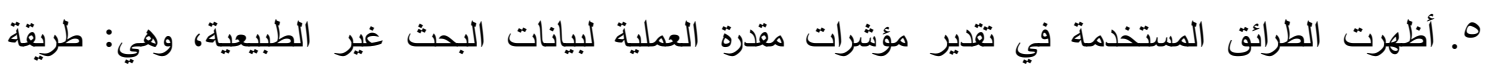
التحويل Box-Cox، وطريقة التباين الموزون، وطريقة Clements، وإيجاد توزيعات أخرى، ومن نتائج الجدول (T)، أن هناك تقارباً في نتائج مؤشرات مقدرة العملية، وأن طريقة Clements هي أفضل الطرائق

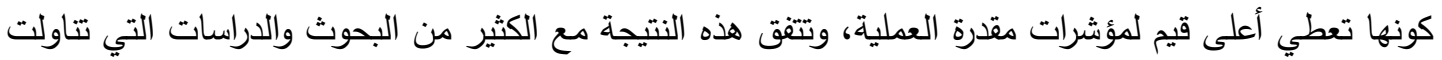
الإقارنة بين نتائج طرائق تقدير مؤشرات مقدرة العملية.

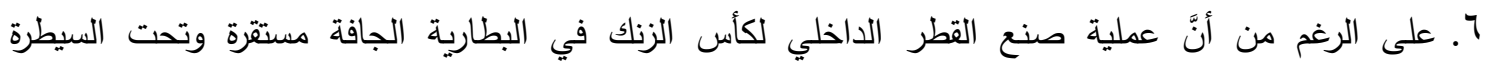
الإحصائية، إلا أنها ليست ذات مقدرة (not capable)، فمن نتائج الجدول (6) تبين أنَّ قيمة مؤشر مقدرة العملية في أفضل أحوالها لم تتجاوز (65\%)، وستكون نسبة البطاريات الجافة المنتجة من المصنع التي هي خارج المواصفات (نسبة المعيب) كبيرة وواقعة بين (3.6\%) و (13.4\%) [انظر الجدول (1)]. وتتفق هذه منه

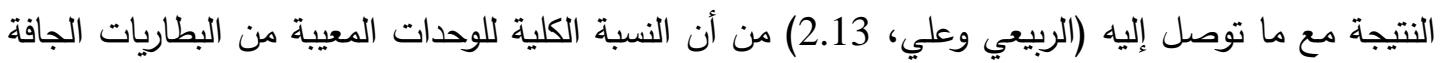
(التي حسبت بطريقة مختلفة عن ما ذكر في هذا البحث) بلغت (28.31\%).

' ـ اعتماد الأساليب الإحصائية في عمل السيطرة النوعية واعتماد اللوحات المناسبة أداةً رقابيةً لتشخيص الانحرافات

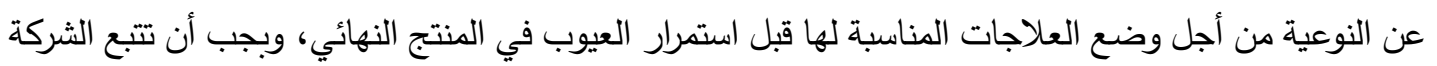
أسلوب حساب مقدرة العملية الإنتاجية أداةً لاختيار المكائن وتثخيصها التي تعتمد على تصنيع أجزاء البطاريات

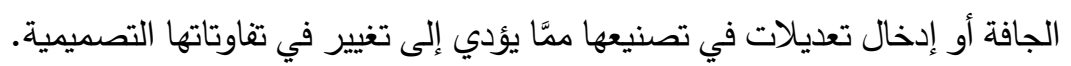

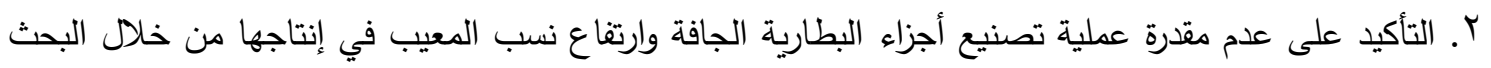
في تتاول مؤشرات مقدرة أخرى وحسابها، وأثير إليها في الفصل الثاني مثل المؤشرات: . $\operatorname{CMA}(\tau, v)$ و r. أشارت الدراسات والبحوث السابقة جميعها التي استند عليها هذا البحث والتي قامت بمقارنة طرائق تقدير

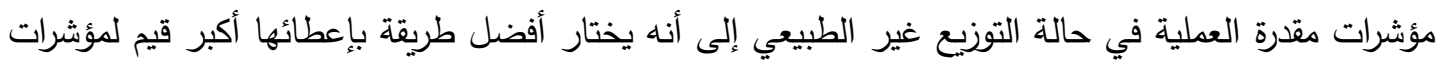

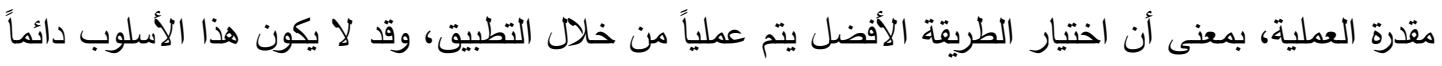

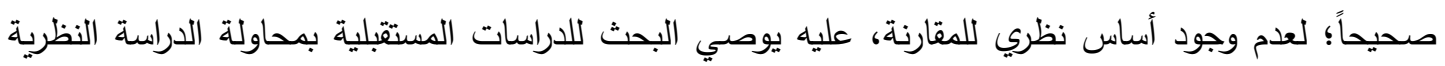
للمقارنة بين الطرائق المذكورة واختيار أفضلها بناءً على بعض المعايير الإحصائية. 


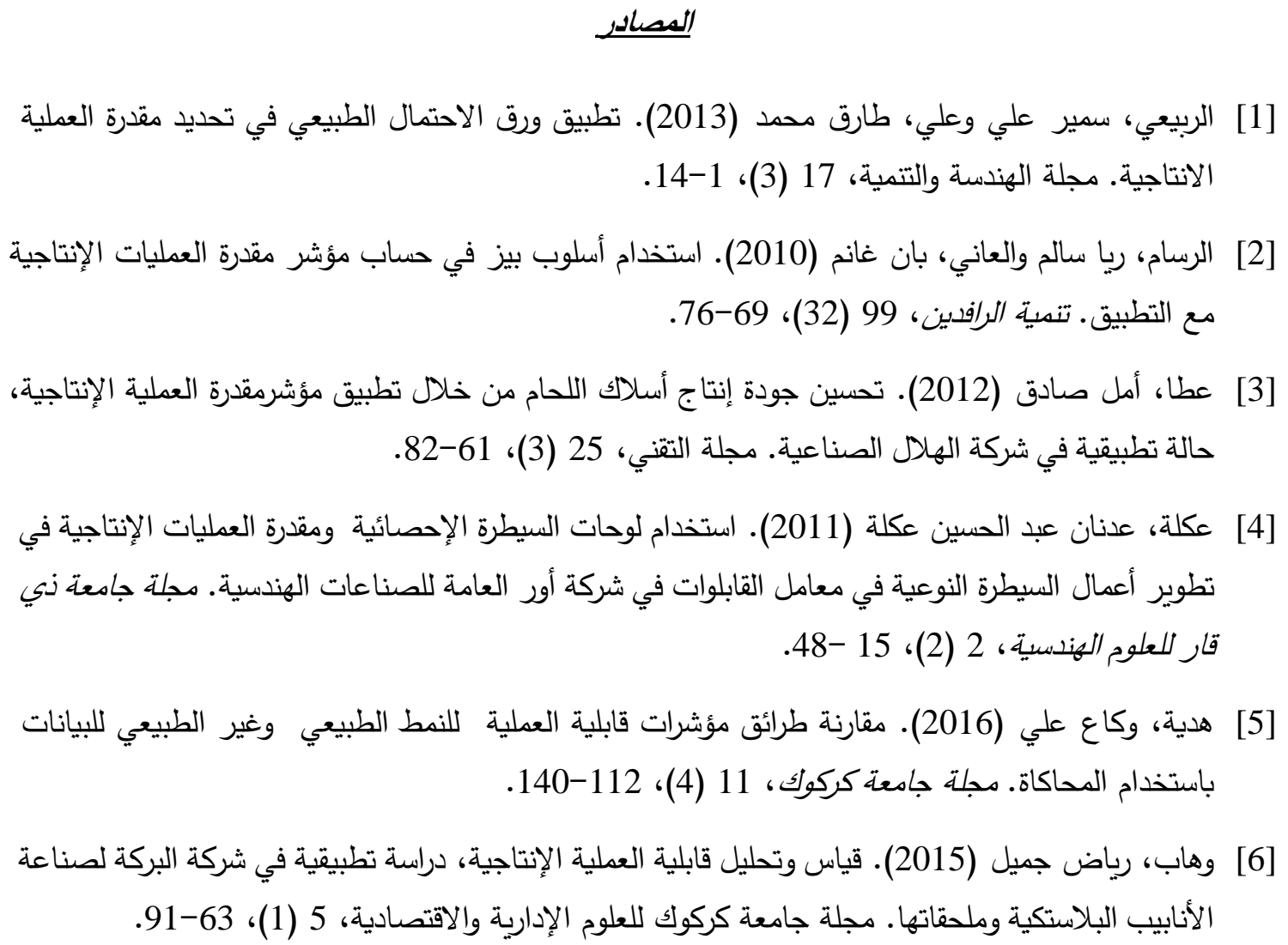

[7] Ahmed, S.; Abdollahian, M. \& Zeephongsekul, P. (2008). Process Capability Estimation for Non-Normal Quality Characteristics: A Comparative of Clements. Burr and Box-Cox Methods. ANZIAM J., 49, 642-665.

[8] Arcidiacono, G. \& Nuzzi, S. (2017). A Review of the Fundamentals on Process Capability, Process Performance, and Process Sigma, and an Introduction to Process Sigma Split. International Journal of Applied Engineering Research, 12 (14), 4556-4570.

[9] Chandra, M.J. (2001). Statistical Quality Control. CRC Press LLC, USA.

[10] Cogollo, S.A. \& Cogollo, J. (2016). Estimating Process Capability Indices Using the Skewed Normal Distribution. Conference Paper, MIPE-407.

[11] Czarski, A. (2009). Analysis of Clements' Method for Capability Indices Estimation. Metallurgy and Foundry Engineering, 35 (1), 55-64.

[12] Elisson, A. (2017). Implementing SPC for non-normal processes with the I-MR chart: A case study. MSc. Thesis, Royal Institute for Technology, Stockholm, Holland.

[13] Kapadia, M. (2020). Measuring Your Process Capability. Symphony Technologies Planning, Design \& Analysis, India.

[14] Korkusuz, D. (2011). Process Capability Analysis for Non-Normal Processes with Lower Specification Limits, MS.c Thesis, Chalmers University of Technology, Gothenburg, Sweden.

[15] Kovarik, M. \& Sarga, L. (2014). Process Capability Indices for Non-Normal Data. WSEAS Transaction on Business and Economics, 11, 419-429. 
[16] Kwiecien, J.W. (2017). Process Capability Calculations with Non-Normal Data in the Medical Device Manufacturing Industry, Ph.D Thesis, Applied Management and Decision Sciences Faculty, Walden University, Minnesota, USA.

[17] Mahmoud, M.A. \& Aufy, S.A. (2013). Process Capability Evaluation for a NonNormally Distributed One. Eng. \& Tech. Journal, 31 (17), 2345-2358.

[18] Montgomery, D. (2013). Introduction to Statistical Quality Control. $7^{\text {th }}$ edition, John Wiley \& Sons, Inc, USA.

[19] Pearn, W.L.; Chen, K.S. \& Lin, G.H. (1999). A generalization of Clements' method for non-normal Pearsonian processes with asymmetric tolerances. International Journal of Quality \& Reliability Management, 16 (5), 507-521.

[20] Selvamuthu, D.D. (2018). Introduction to Statistical Methods, Design of Experiments and Statistical Quality Control. Springer Nature Singapore Pte Ltd.

[21] Senvar, O. \& Sennaroglu, B. (2016). Comparing performances of clements, BoxCox, Johnson methods with Weibull distributions for assessing process capability. Journal of Industrial Engineering and Management (JIEM), 9 (3), 634-656.

[22] Shore, H. (1998). A New Approach to Analyzing Non-normal Quality Data with Application to Process Capability Analysis. International Journal of Production Research, 36 (7), 1917-1933.

[23] Wooluru, Y.; Swamy, D.R. \& Nagesh, P. (2015). Process Capability Estimation for Non-Normality Distribution Data using Robust Methods - A Comparative Study. International Journal for Quality Research, 10 (2), 407-420. 